\section{Application of Biasing Techniques to the Contributon Monte Carlo Method}

\section{DR. 763}

MASTER 
APPLICATION OF BIASING TECHNIQUES TO THE

CONTRIBUTON MONTE CARLO METHOD

by

A. Dubi

S. A. W. Gerstl

ABSTRACT

Recently, a new Monte Carlo Method called the "Contributon Monte Carlo Method" was developed. The method is based on the theory of "contributons" and utilizes a new recipe for estimating target responses using a volume integral over the contributon current. The analog features of the new method were discussed in previous publications. In the following, we examine the application of sowe biasing methods to the new contributon scheme. A theoretical model is developed that enables an analytic prediction of the benefit to be expected when these biasing schemes are applied to both the contributon method and regular Monte Carlo. This model is verified by a variety of numerical experiments and is shown to yield satisfying results, especially for deeppenetration problems. Other considerations regarding the efficient use of the new method are also discussed and remarks are made as to the application of other biasing methods.

\section{INTRODUCTION}

We first briefly review the basics of the contributon Monte Carlo method. Consider the situation of a typical radiation transport problem. A neutron source $Q(\bar{p})$ is distributed over a given source region, then a detector, described by a given response function $R(\bar{p})$, is given such that there is no overlap between 
the detector region and the source region. Between the source and the detector we have the medium through which particles are traasported. The classical recipe to calculate a desired detector response, $I$, uses an integral of the product of the neutron flux and the detector response function over the detecior region $V_{R}$ and all $0^{+}$her phase-space variables,

$$
I=\iiint_{V_{\bar{h}}^{\prime}} \phi(\bar{p}) \cdot R(\bar{p}) d \bar{r} d E d \bar{\Omega} \equiv\langle\phi, R\rangle,
$$

where $\phi(\vec{p})$ is the neutron flux at the phase space point $\vec{p}=(\bar{r}, E, \bar{\Omega})$. All forward (in contrast to adjoint) Monte Carlo methods are based on evaluating Eq. (1); we will refer to such techniques as regular Monte Carlo. Basically, in regular Monte Carlo one obtains information about the product of $\phi(\overline{\mathrm{p}}) \mathbf{R}(\overline{\mathrm{p}})$ by sinulating the transport of the particles from the source region to the detector region using either analog or nonanalog transport kernels with proper weighting.

A second common recipe, equivalent to Eq. (1), uses the integral of the product of the adjoint flux $\phi^{+}(\bar{p})$ and the source distribution function $Q(\bar{p})$ over the source region $v_{Q}$ and all other phase-space variables,

$$
I=\iiint_{V_{Q}} \phi^{+}(\bar{p}) Q(\bar{p}) \mathrm{d} \dot{r} \mathrm{dE} \overline{\mathrm{\Omega}} \equiv\left\langle\phi^{+}, Q\right\rangle
$$

In contrast to Eq. (1), Eq. (2) requires an adjoint transport calculation to determine $\phi^{+}(\bar{p})$. A less commonly used third recipe is obtained $(1)$ by integrating the current of "contributons", defined as the adjoint weighted neutron current, over a closed surface, F, enclosing either the source or the detector region,

$$
I=\iiint_{F}(\bar{n} \cdot \bar{\Omega}) \phi(\bar{p}) \phi^{+}(\bar{p}) d F d E d \bar{\Omega} \equiv \iiint_{F}(\bar{n} \cdot \bar{\Omega}) \psi(\bar{p}) d F d E d \bar{\Omega},
$$


where $\bar{n}$ is a unit vector perpendicular to the surface $F$. However, the use of Eq. (3) requires the knowledge of both, $\phi(\bar{p})$ as well as $\phi^{+}(\bar{p})$, which way be obtained from separate forward and ajoint simulations. Deutsch ${ }^{5}$ has shown that it is possible to evaluate Eq. (3) from a forward simulation alone, but this schene basically reverts to the first recipe as identified by Eq. (1).

Yet another recipe, which is the basis for the "contributon Honte Carlo Method", is obtained by transforming the surface integral in Eq. (3) to a volume integral ${ }^{3}$. This volume is not arbitrary but is defined by integrating Eq. (3) with respect to an additional coordinate $\alpha$ perpendicular to $F$ such that for any fixed value of $\alpha$ the surface integral yields $I$. Thus, the integration over $\alpha$ simply yields the lateral dimension of the volume and we may write

$I=\frac{1}{\Delta} \iiint_{F(\alpha)} \int_{\alpha}^{\alpha_{2}}(\bar{n} \cdot \bar{\Omega}) \phi(\bar{p}) \phi^{+}(\bar{p}) d F d \alpha d E d \bar{\Omega}=\frac{1}{\Delta} \iiint_{V}(\bar{n} \cdot \bar{\Omega}) \phi(\bar{p}) \phi^{\nu}(\bar{p}) d V d E d \bar{\Omega}$,

where $\Delta=\alpha_{2}-\alpha_{1}$ is the lateral dimension of the volune $v$. Note that this volume of integration may actually be constructed fron a continuous set of parallel surfaces to all of which Eq. (3) applies, thus both $\Delta$ and $\bar{n}$ are well defined. We now describe the Monte Carlo procedure for an unbiased estimation of I via the new recipe of $\mathrm{Eq}$. (4). The rigorous proof of this being an unbiased estimator is given in Ref. 3. The estimation is done in the following steps:

a. A source particle, which is also referred to as primary particle, is enitted and its path followed to a collision point $\bar{p}_{i}=\left(\bar{r}_{i}, \bar{\Omega}_{i}, E_{i}\right)$ inside $V$, where $\bar{\Omega}_{i}$ and $E_{i}$ are the direction and energy of the incoming particle. Every collision gives us an estimate for the collision rate $\Sigma_{t} \phi\left(\bar{p}_{i}\right)$, and thus for the particle flux at $\bar{p}_{i}$.

b. At every collision point $\bar{r}_{i}$ inside $V$ a secondary particle is generated with probability $\left|\bar{\Omega}_{i} \cdot \bar{n}\right|$ in direction $\bar{\Omega}_{i}$ and with energy $E_{i}$. The secondary's path is followed until it scores in the detector, in which case an estinate for the importance, i.e., the adjoint flux at $\dot{p}_{i}$, is obtained. The score of the secondary is taken positive or negative depending on the $\bar{\Omega}_{\dot{i}} \cdot \vec{n}$ being positive or negative. $*$ 
c. The total score for each primary is then given by the sum of the scores of all the secondaries produced along the primary's track where each score is divided by the total cross section at the collision point at which the secondary was generated.

d. The statistical average of the scores due to all primaries divided by $\Delta$ yields then an unbiased estimate of $\mathrm{I}$, according to $\mathrm{E}_{\mathfrak{1}}$. (4).

In the terminology of "contributons", the secondary particle serves the purpose of identifying the primary that produced it, as a contributon if the secondary scores in the detector. The statistical averaging over all primaries that produced scoring secondaries is equivalent to integrating over the contributon current that passed through the volume $V$.

It is worthwhile at this point to make a very important observation. The contributon method described above is not a new biasing scheme, it employs however a new recipe to calculate detector responses, i.e., Eq. (4). Therefore, any biasing scheme that may be incorporated into analog regular Monte Carlo can be also incorporated in exactly the same manner into the analog "contributon" Monte Carlo as will be proven later. Another interesting feature is the following: Since secondaries are produced with probability $|\bar{n} \cdot \bar{\Omega}|$ one has some control over the production of secondaries via a proper choice of the volume of integration, or the surfaces fron which this volume is constructed. It is alkays possible to chose the volume such that secondaries will be produced with greater probability in direction towards the detector. This capability can be viewed as a type of "natural" angular biasing without introducing any weights. Note also that the transport of the source particles (primaries) is not affected at all by the fact that $s$ ccondaries are produced. This feature is in contrast to the case of splitting in which the production of a secondary causes a reduction in the weight of the splitted particle. The nost important consequence of this fact is that the implementation of the contributon method in any existing Monte Carlo transport code requires no major interferance in the code. Moreover, since the primary particle transport is unperturbed it can yield additional information by regular Monte Carlo scoring. This additional information may be combined with the estiate from the contributon method to improve the overall statistics.

FIn the scheme described in Ref. 3 , the quantity $(\bar{n} \cdot \bar{\Omega})$ is attached to the score rather than to the sampling; here, however, the $|\bar{n} \cdot \bar{\Omega}|$ is moved to the sampling process and only its sign is left with the score. 
The above mentioned features will be demonstrated and further discussed later in this paper. In Section II we demonstrate how the contributon Monte Carlo procedure can be derived rigorously with the methods of variational analysis. Sections III and IV develop a simple formula which allows the potential user of the contributon Monte Carlo method to predict quantitatively what computational benefit (if any) might be expected for a given problem compared to regular Monte Caclo if the same biasing paraneters are assumed for both methods. The remaining sections demonstrate with a large number of sample calculations the validity of the theoretical developments and identify the accuracy of the predicted benefits.

\section{VARIATIONAL DERIVATION OF CONTRIBUTON MONTE CARLO}

Our objective in the theory of contributons is to identify equations cr procedures which allow us to compute $\psi=\phi(\bar{p}) \phi^{+}(\bar{p})$ in the volume $V$ from first principles and then calculate I via Eq. (4). This objective may be reformulated 6 into a classic variational problem of rendering the functional I stationary, treating the forwarc transport equation $H \phi=Q$ and the adjoint transport equation $\mathrm{H}^{+} \phi^{+}=\mathrm{R}$ as constraints on $\phi$ and $\phi^{+}$. Using Eq. (4) for the procedure to calculate $I$ we are led to consider the functional

$$
\left.F\left[\tilde{\phi}, \tilde{\phi}^{+}, \tilde{x}, \tilde{\chi}^{+}\right]=\frac{1}{\Delta}\left\langle(\bar{n} \cdot \bar{\Omega}) \tilde{\phi}^{+}, \theta_{v}\right\rangle+\left\langle\tilde{\chi}, R-H^{+} \hat{\phi}^{+}\right\rangle+\tilde{\chi} \tilde{\chi}, Q-H \tilde{\phi}\right\rangle,
$$

where $\tilde{\phi}, \tilde{\phi}^{+}$are trial functions for $\phi$ and $\phi^{+}$, respectively; $\tilde{X}$ and $\tilde{\chi}^{+}$are Lagrange multipliers and $\theta_{v}$ is a spatial step function which is 1 inside the volume $v$ and zero outside. Performing a variation SF in Eq. (5) by varying $\tilde{\phi}, \hat{\phi}^{+}, \tilde{x}$ and $\tilde{\chi}^{+}$ we obtain

$$
\begin{aligned}
& \delta F=\frac{1}{\Delta} \underbrace{\left\langle\underline{(n \cdot S) \phi^{+} \delta \phi, \Theta}\right\rangle}+\frac{1}{\Delta}\langle\underbrace{\left.(n \cdot \Omega) \phi \delta \phi^{+}, \theta\right\rangle} \\
& \left\langle\delta \phi,(n \cdot \Omega) \phi^{+} \Theta>\quad\left\langle\delta \phi^{+},(\underline{n} \cdot \Omega) \phi \Theta>\right.\right.
\end{aligned}
$$




$$
\begin{array}{r}
+\left\langle\delta \chi, R-H^{+} \phi^{+}\right\rangle-\underbrace{\frac{\left\langle\chi, \delta\left(H^{+} \phi^{+}\right)\right.}{H^{+} \delta \phi^{+}}}_{\left\langle H \chi, \delta \phi^{+}\right\rangle} \\
+\left\langle\delta \chi^{+}, \mathrm{Q}-\mathrm{R} \phi\right\rangle-\underbrace{\frac{\left\langle\chi^{+}, \delta(H \phi)\right\rangle}{H \delta \phi}}_{\left\langle H^{+} \chi^{+}, \delta \phi\right\rangle}
\end{array}
$$

Requiring $\delta F=0$ for arbitrary variations $\delta \tilde{\phi}, \delta \hat{\phi}^{++}, \delta \tilde{x}, \delta \tilde{x}{ }^{+}$yields the four Euler equations for the stationary values $\phi, \phi^{+}$of our trial functions $\tilde{\phi}, \tilde{\phi}^{+}$ana for the stationary values $x, \chi^{+}$of the $\bar{L}$ àzrange Multipliers $\tilde{\chi}, \tilde{x}^{+}$:
A) $\mathbf{H} \phi=Q$,
B) $H^{+} \phi^{+}=R$,
C) $\mathrm{HX}=\frac{1}{\Delta}(\vec{n} \cdot \vec{\Omega}) \phi \Theta_{\mathrm{v}}$,
D) $\mathrm{H}^{+} \mathrm{X}^{+}=\frac{1}{\Delta}(\overrightarrow{\mathrm{n}} \cdot \vec{\Omega}) \phi^{+} \theta_{\mathrm{v}}$.

Eqs. (6A) and (6B) are, of course, the forward and adjoint Boltzmann equations which were incorporated in the functional (5) already as constraints. However, Eqs. (6C) and (6D) are new and identify equations for the Lagrange Multipliers. In order to obtain a physical interpretation for $X$ and $X^{+}$we nultiply Eq. (6C) with $\phi^{+}$and integrate over all phase space.

$$
\left\langle\phi^{+}, H \chi\right\rangle=\frac{1}{\Delta}\left\langle(\vec{n} \cdot \vec{\Omega}) \phi \phi^{+}, \theta_{v}\right\rangle=I
$$

While the right-hand side of Eq. (7) equals I according to Eq. (4), the lefthand side can be easily transformed using the definition of the adjoint operator $\mathrm{H}^{+},\left\langle\phi^{+}, \mathrm{HX}\right\rangle=\left\langle\mathrm{H}^{+} \phi^{+}, X\right\rangle$, and replacing $\mathrm{H}^{+} \phi^{+}$with $\mathrm{R}$ according to the adjoint Boltzmann equation (6B), to obtain from Eq. (7) 


$$
I=\langle\mathbf{R}, X\rangle
$$

Eq. $(\varepsilon)$ is yet another recipe to calculate $I$, and it identifies the Lagrange Multiplier $X$ as a new flux distribution function.

In fact, if we consider Eqs. (6A), (6C) and (8) only, the following deterministic approach to compute $I$ is indicated:

1. Solve Eq. (6A) to obtain $\phi$ in $V$,

2. construct the source term $(\bar{n} \cdot \bar{\Omega}) \phi$ in $V$ for $E q .(6 C)$,

3. solve $\mathrm{Eq}$. $(6 \mathrm{C})$ to obtain $\chi$ in the detector region, and

4. calculate I fron $\mathrm{Eq}$. (8).

Accurdingiy, $\phi$ may be called a primary flux distribution, $\chi$ a secondary particíe fiux, and the above procedure is recognized as a "bootstrapping" technique which has been used in the past in some severe deep-penetration shielding calculations where it appeared intuitively advantageous to subdivide the spatial domain into two or more overlapping parts (7). In fact, the classical "bootstrapping" always used a surface source for the secondaries rather than a volumetric source as specified in Eq. (6C). Since this procedure requires the solution of two transport equations, its advantages in efficiency versus the conventional methods to compute I. are questionable. However, we can also visualize this "bootstrapping" technique for each individual source particle and obtain the following Monte Carlo procedures ${ }^{3}$ :

1. Follow a source particle (primary) into the volume $V$ where it may make $k$ collisions, giving an estimate of $\phi$ at these collision points.

2. Start at each collision in $V$ a secondary particle with probability $|\bar{n} \cdot \bar{\Omega}|$ in the same direction as the primary entered the collision.

3. Score each secondary's contribution to $I$ as $\pm R_{i}$ at the detector location, where the sign depends upon $(\vec{n} \cdot \vec{\Omega}) \geqslant 0$ in step 2 .

4. Then the score for each primary is the sum over all its secondaries' scores $\sum_{i=1}^{k} \pm R_{i}$.

Step 1 corresponds to solving Eq. (6A) and in step (2) the source term to Eq. (6C) is prepared. Following the secondaries to the detector corresponds to solving Eq. (6C) while steps 3 and 4 are equivalent to calculating the integral 
of Eq. (8). The technical difference between the "deterministic bootstrapping" using Eqs. (6A) and (6C) and the above-described "Monte Carlo bootstrapping" is the complete correlation between the primary paricle's direction and energy $(\bar{\Omega}, E)$ at the collision point $\bar{r}_{k}$ and the starting direction and energy of the secondary which is explicitely taken into account in the M.C. procedure but not in the deterministic bootstrapping procedure. Eqs. (6B) and (6D) define a corresponding adjoint contributon M.C. procedure.

III. A SEMI-INTUITIVE MODEL FOR THE BENEFIT FACTOR

In the following the implementation of exponential path length stretching in particular, and other biasing techniques in general, in the contributon method is considered. In the exponential path stretching, the total cross section $\Sigma_{t}$ is replaced by a reduced total cross section $\Sigma_{t}^{*}=\Sigma_{t}(1-p)$ where $0 \leq p<1$. As a result the path length from one collision to another is sampled from a probability density $g_{p}(x)=\Sigma_{t}^{*} e^{-\Sigma_{t}^{*} x}$ rather than from the analog density $f(x)=\Sigma_{t} e^{-\Sigma_{t} x}$. In addition at the end of each free flight the weight of the particle is multiplied by

$$
f(x) / g_{p}(x)=e^{-p \Sigma t^{x}} /(1-p)
$$

to account for the distortion in the free flight kernel.* In general, the stretching parameter $p$ may have some angular dependence. In addition to the path stretching, we consider weighting in lieu of absorption, where particles are not allowed to be absorbed; instead, upon collision, the weight of the particle is mutiplied by the scattering probability $\Sigma_{s} / \Sigma_{t}$. This is nost useful in transmission problems to which our main attention is directed.

The efficiency of a given method is measured by the quantity $1 / 9=\sigma^{2} \cdot \tau$ where $\sigma^{2}$ is the variance and $\tau$ is the average time needed to follow one source particle history. If we denote the efficiency factors of the contributon method and regular Monte Carlo by $1 / q_{c}$ and $1 / q_{R}$, respectively, then a benefit factor may

FIf the flight ends at a vacuum boundary the weight is multiplied by $e^{-\Sigma_{t} p x}$ only. 
be defined by

$$
B=\frac{q_{c}}{q_{R}}=\frac{\left(\sigma^{2} \cdot \tau\right)_{R}}{\left(\sigma^{2} \cdot \tau\right)_{C}}
$$

In the following we develop a theoretical model to predict $B$ for the case where the same exponential biasing is applied to both, the regular M.C. and the contributon M.C. Specifically, our goal is to present the second moment for the contributon method and regular Monte Carlo in a form which will enable a quantitative comparison.

We first formalize the situation for regular Monte Carlo. Each source particie having a rardom walk $\left(\vec{p}_{0}, \bar{p}_{1} \ldots \bar{p}_{n}\right)$ [where $p_{0}$ is the source evission point, with $\bar{p}=(\bar{r}, \bar{\Omega})$, where $\bar{\Omega}$ is the direction with which the particle goes into a collision point $\overline{\mathbf{r}}]$ scores a weight given by $w_{p}\left(\bar{p}_{0}, \bar{p}_{1} \ldots \bar{p}_{n}\right)$. This score includes both the accumulated weight of the particle along the track and the specific estinator scoring. The probability density function for the above track may be expressed in the notation of Ref. (8) as

$f_{p}^{*}\left(\tilde{p}_{0}, \bar{p}_{1} \ldots \bar{p}_{n}\right)=\theta^{*}\left(\bar{\Omega}_{1}\right) \quad T^{*}\left(\vec{\Omega}_{1}: \bar{r}_{0} \rightarrow \bar{r}_{1}\right) c^{*}\left(\vec{r}_{1}: \bar{\Omega}_{1} \rightarrow \bar{\Omega}_{2}\right) \ldots T^{*}\left(\bar{\Omega}_{n}: \bar{r}_{n-1}+\bar{r}_{n}\right) p^{*}\left(\bar{p}_{n}\right)$,

where

$$
\begin{aligned}
& \theta^{*}(\bar{\Omega}) \text { - is the biased source angular density, } \\
& T^{*}-\text { is the biased free flight kernel, } \\
& C^{*}-\text { is the biased collision kernel, } \\
& p^{*}\left(\bar{p}_{n}\right) \text { - is the biased absorption probability. }
\end{aligned}
$$

The average weight (which is the desired target response I) is then given by averaging all the scores $w_{p}$ over all possible tracks in the form 


$$
I=\left\langle w_{p}\right\rangle=\sum_{n=1}^{\infty} \int \ldots \int w_{p}\left(\bar{p}_{0} \ldots \bar{p}_{n}\right) f^{*}\left(\bar{p}_{0} \ldots \bar{p}_{n}\right) d \bar{p}_{1} \ldots d \bar{p}_{n} .
$$

The second moraent is then given by

$$
s_{R}^{2}=\left\langle w_{p}^{2}\right\rangle=\sum_{n=1}^{\infty} \int \ldots \int w_{p}^{2}\left(\bar{p}_{0} \ldots \bar{p}_{n}\right) f^{*}\left(\bar{p}_{0} \ldots \bar{p}_{n}\right) d \bar{p}_{1} \ldots d \bar{p}_{n}
$$

In the contributon wethod the randon walk of a secondary may be viewed as starting from the source in the following way $\left(\bar{p}_{0} \bar{p}_{1} \ldots \bar{p}_{i} \bar{p}_{1} \bar{p}_{2} \cdots \cdot \bar{p}_{k}\right)$ where the $\bar{p}_{i}$ are collision points of the primary at which a secondary was produced. What this anounts to is looking at the track of a secondary as starting from the actual source where that part of the track of the primary prior to the emission of the secondary is considered as a portion of the secondary's track. Note that the secondary's track described above has $\bar{r}_{i-1} \bar{r}_{i}$ and $\bar{r}_{1}^{i}$ colinear, and the secondary made k-1 collsions*. Now instead of averaging over primary particles, as is suggested in Eq. (1) we can average formally over the secondaries according to Eq. (8); i.e. we can ask what is the score attached to a secondary track of the form presented above and what is the probability density attached to those scores, and then take the average. This will indeed yield the same result as if the scores were averaged over the source particles, but it will give more comprehensive results when we look at the second moment. Before doing so formally, we describe how this is done in a semi-intuitive manner.

Consider the situation presented in Fig. 1. Shown there is a particle track from the source through a slab-shaped homogeneous medium to the detector (bing in this case the vacuum boundary at $z=z_{2}$ ). In Regular Monte Car?o the probability density and the score of that track are given by Eq. (10) and by $w_{p}$, respectively. Suppose now that the volume of integration is that between the surfaces $z=z_{1}$ and $z=z_{2}$. We may ask first what will be the probability density to obtain a secondary's track like the one shown. Note, that not only one secondary may follow this track, but there are many secondaries that can be generated from primaries along that track (in $z_{1}<z<z_{2}$ ) and then follow the track while the primary takes another course somewhere in the mediur. Three such

$*_{\text {where }} \bar{p}_{k}^{i}$ is the point of transmission through a surface. 


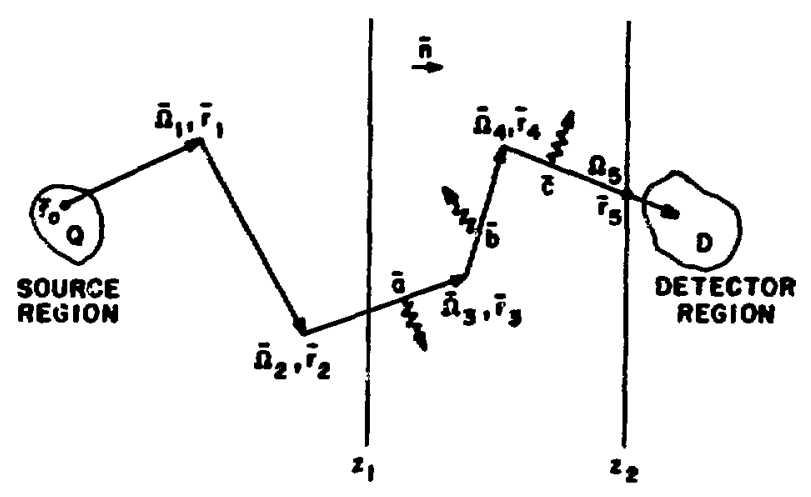

Fig. 1. Typical particle track.

events are shwon in Fig. 1 where the curved arrow represents the primary. That is, we can visualize a primary following the track up to point $\bar{a}$, colliding there, changing direction, and going into the medium. However the secondary produced there (with probability $\left.\mid \bar{\Omega}_{3} \cdot \bar{n} !\right)$ continues in a straight line to $\bar{p}_{3}$, etc. The same may be repeated at points $\bar{b}$ and $\bar{c}$ and indeed on every collision point continuously along the track within the volume of integration. Let us now write down the probability density for the event just described at point $\bar{a}$; this is

$$
\begin{aligned}
& \mathrm{f}_{\mathrm{a}}\left(\overline{\mathrm{p}}_{\mathrm{o}}, \overline{\mathrm{p}}_{1}, \overline{\mathrm{p}}_{2}, \ddot{\mathrm{a}}, \overline{\mathrm{p}}_{3}, \ldots \overline{\mathrm{p}}_{5}\right)=\mathrm{Q}^{*}\left(\bar{\Omega}_{1}\right) \mathrm{T}^{*}\left(\tilde{\Omega}_{1} ; \overline{\mathrm{r}}_{\mathrm{o}} \rightarrow \overline{\mathrm{r}}_{1}\right) \mathrm{C}^{*}\left(\overline{\mathrm{r}}_{1} ; \bar{\Omega}_{1}+\bar{\Omega}_{2}\right) \mathrm{T}^{*}\left(\bar{\Omega}_{2} ; \overline{\mathrm{r}}_{1} \rightarrow \overline{\mathrm{r}}_{2}\right) \\
& \mathrm{c}_{3}^{*}\left(\overline{\mathrm{r}}_{2} ; \bar{\Omega}_{2} \rightarrow \bar{\Omega}_{3}\right) \cdot \mathrm{T}^{*}\left(\bar{\Omega}_{3} ; \overline{\mathrm{r}}_{2}+\overline{\mathrm{a}}\right) \mathrm{T}^{*}\left(\bar{\Omega}_{3} ; \overrightarrow{\mathrm{a}} \rightarrow \overline{\mathrm{r}}_{3}\right) \cdot \mathrm{c}^{*}\left(\overline{\mathrm{r}}_{3} ; \Omega_{3} \bar{z}_{2}\right) \ldots \ldots . \times \mathrm{x}\left|\overline{\mathrm{n}} \cdot \bar{\Omega}_{3}\right| \cdot
\end{aligned}
$$

The free flight kernel $T\left(\bar{\Omega} ; \bar{r}^{\prime} \bar{r}^{\prime}\right)$ describes the probability density that a particle that was emitted at $\bar{r}$ in direction $\bar{\Omega}$ will enter its next collision at $\bar{r}$ ' given that $\bar{r}$ are $\bar{r}$ ' are colinear. In a homogenous medium, for exponential path 
stretching, $T$ will take the folii

$$
\mathrm{T}\left(\bar{\Omega} ; \overline{\mathrm{r}} \rightarrow \overline{\mathrm{r}}^{\prime}\right)=\Sigma \mathrm{t}^{*} \mathrm{e}^{-\Sigma_{\mathrm{t}}^{*}\left|\overline{\mathrm{r}}-\overline{\mathrm{r}}^{\prime}\right|} .
$$

Since $\bar{r}_{2}$, $\bar{a}$ and $\bar{r}_{3}$ are coligear it is easy to show that

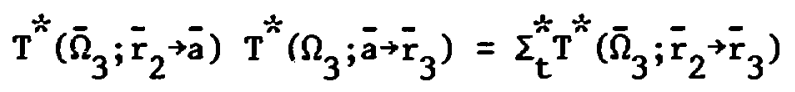

Thus, $T$ is independent of $\bar{a}$. (A relation similar to the above can be obtained for all biased free flight kernels where the dependence of the kernel on the path length is exponential). To obtain the probability density for the track $\left(\overline{\mathrm{p}}_{1}, \overline{\mathrm{p}}_{2}\right.$, $\overline{\mathrm{p}}_{3}, \overline{\mathrm{p}}_{4}, \overline{\mathrm{p}}_{5}$ ) in the contributon scheme we need then to integrate Eq. (13) over that portion of the track which lies within the volumn if integration. In view of the fact that $f_{a}$ is independent of $a^{-*}$, the integration yields immediately

$$
\begin{aligned}
& f_{c}\left(\bar{p}_{1} \ldots \bar{p}_{5}\right)=\left\{Q^{*}\left(\bar{\Omega}_{1}\right) T^{*}\left(\bar{\Omega}_{1} ; \bar{r}^{*} \bar{r}_{1}\right) \ldots . c^{*}\left(\bar{r}_{4} ; \bar{\Omega}_{4} \rightarrow \bar{\Omega}_{5}\right) \quad T\left(\bar{\Omega}_{5} ; \bar{r}_{4} \rightarrow \bar{r}_{5}\right) / \Sigma_{t}^{*}\left(\bar{r}_{5}\right)\right\} \\
& x\left[\left|\overline{\Omega_{i}} \cdot \overline{\mathrm{n}}\right| \int_{0}^{\left|\bar{r}_{3}-\bar{x}_{2}\right|} \mathrm{da}+\left|\bar{\Omega}_{4} \cdot \overline{\mathrm{n}}\right| \int_{0}^{\left|\bar{r}_{4}-\bar{r}_{3}\right|} \mathrm{da}+\left|\bar{\Omega}_{5} \cdot \overline{\mathrm{n}}\right| \int_{0}^{\left|\bar{r}_{5}-\overline{\mathrm{r}}_{4}\right|} \mathrm{da}\right] \cdot \Sigma^{*} \mathrm{t}
\end{aligned}
$$

Note that the product in the curiy brackets is identical to the proability density $f_{p}$ of the same track in regular Monte Carlo when the same biasing is applied. The sum in the square brackets simply adds up to $z_{2}-z_{1}=\Delta$ which is the lateral dimensical of the volume of integration (because each term in the sum yields the trajectory of the corresponding path length segment in the n-direction). With these results we can write down the relation between the

$*$ We have assumed $\Sigma_{t}$ independent of energy 
probability density for a given track in the contributon schene, $f_{c}$, and in the regular scheme, $f_{p}$, as

$$
f_{c}\left(\tilde{p}_{1} \ldots \bar{p}_{n}\right)=\Delta \cdot \Sigma_{t}^{*} f_{p}\left(\bar{p}_{1}, \ldots \cdot \bar{p}_{n}\right)
$$

This relation will be proven in a formal manner in the following paragraph. It is important, however, at this point to note the conditions for the vailidity of Eq. (14). This relation will not hold for those tracks in which any $\left(\bar{\Omega}_{i} \bar{n}\right)$ will be negative. In such cases the sum of trajectories will be larger than $\Delta$. The relation in Eq. (14) is valid for tracks which originated at the source and terminated at the detector. Consider now the weight scored by the track in Fig. 1: Limiting ourselves to the cases wher zcoring (using any estimator) is made only at the detector region, we may noif tr:t the weight scored by each secondary will be equal to the weight scores by any other secondary following the same track (which was, however, generated elsewhere on the track). (This again dc's not include secondaries that where produced on segments of the track for which $\bar{\Omega}_{i} \bar{n}<0$. Such secondaries will have the same absolute weight scored but with a negative sign). This weight will also be equal to the weight scored by the same path in regular Monte Carlo excipt for the fact that an additional collision point somewhere along the path has to be considered for the secondary in the contributon scheme. Thus the relaiion between the weight scored by a secondary in the contributon scheme and a particle in the regular scheme is given by

$$
w\left(\bar{p}_{1} \ldots \bar{p}\right)=\frac{w_{p}\left(\bar{p}_{1} \ldots \bar{p}_{n}\right)}{\sum_{t}^{t} \Delta}
$$

With the additional approximation of neglecting the correlation between secondary particles generated from the same primary we can write the second moment of the contributon scheme, analogous to $\mathrm{Eq}$. (12), as 


$$
s_{c}^{2}=\left\langle w_{c}^{2}\right\rangle=\sum_{n=1}^{\infty} \int \ldots \int w_{c}^{2}\left(\bar{p}_{1} \ldots \bar{p}_{n}\right) f_{c}\left(\bar{p}_{1} \ldots \bar{p}_{n}\right) d \bar{p}_{1} \ldots d \bar{p}_{n},
$$

and substituting the relations we derived between $w_{p},{ }^{\prime} c, f_{p}$ and $f_{c}$, we obtain the relation

$$
s_{c}^{2}=\frac{s_{R}^{2}}{\Delta \Sigma_{t}^{\frac{1}{t}}}
$$

The above discussion is included, both to make the approach to the theoretical proof, which follows, clearer and to stress the importance of the fact that in th contributon scheme all secondaries following the same path (in a homogeneous medium) will have exactly the same weight to score. This feature is unique to this scheme. It is this property of the contributon scheme that enables the development of a theoretical comparison between regular Monte Carlo and the contributon Monte Carlo.

IV. FORMAL DERIVATION OF THE BENEFIT FACTOR

Let us first write down the exact form of the second moment in the contributon scheme. Before doing that we recall that $f^{*}\left(p, \bar{p}_{1}, \ldots, \bar{p}_{n}\right)$ is the probability density function for a track of a particle that was emitted at $\bar{p}$, made its first collision at $\bar{p}_{1}$ and was absorbed at $\bar{p}_{n}$. Note also the normalization condition

$$
\sum_{n=1}^{\infty} \int \ldots \int f^{\star}\left(\bar{p}, \bar{p}_{1}, \ldots . \bar{p}_{n}\right) d p_{1} \ldots d p_{n}=1
$$

which means that an emitted particle will eventually be absorbed with probability one. 
We may now write the second ir.went in the form

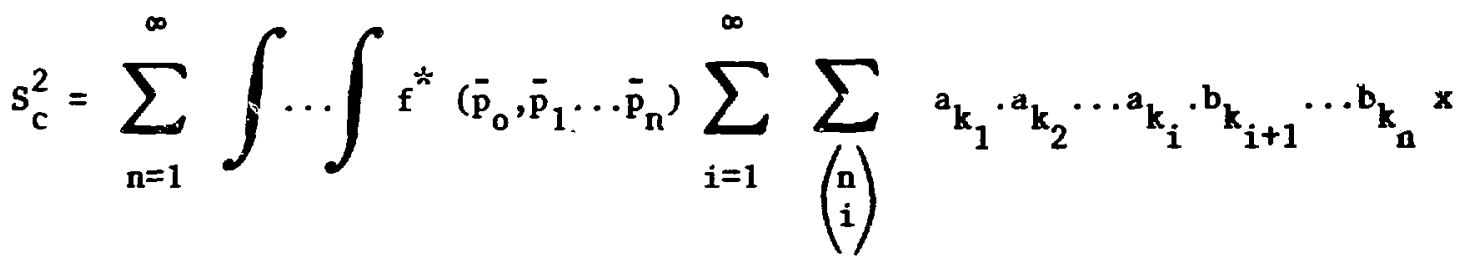

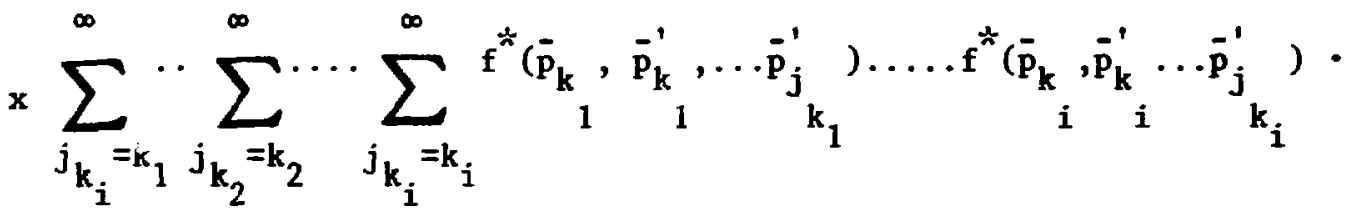$$
\left(w_{c_{k_{1}}}+{ }^{w} c_{k_{2}}+\ldots w_{c_{k_{i}}}\right)^{2} d \bar{p}_{0} \ldots d \bar{p}_{n} d \bar{p}_{k_{1}}^{\prime} \ldots d \bar{p}_{j_{k_{1}}} \ldots d \bar{p}_{k_{i}}^{\prime} \ldots d \bar{p}_{j_{k_{i}}}
$$

In Eq. (19) we mutiply the square of the socre of an event by the probability density of that event and then integrate, and sum, over a continuum of independent events that may accur in the contributon transport. To facilitate understanding of Eq. (19) consider a specific event in the following way: A primary particle makes $n$ collisions and is absorbed upon the $n^{\prime}$ th collision. The collision points are $\left(\bar{p}_{1}, \bar{p}_{2}, \ldots \bar{p}_{n}\right)$. (The n'th collision point may be a vacuum boundary in which case the total cross section at $\left(\bar{p}_{n}\right)$ is infinite and the absorption probability is unity). From these $n$ collision points $i(\leq n)$ secondaries are generated at specific collision points $\left(\bar{p}_{k_{1}}, \bar{p}_{k_{2}} \ldots \bar{p}_{k_{1}}\right)$. For each given $i$ there are $\left(\begin{array}{l}n \\ i\end{array}\right)$ different specific choices of $\left(k_{1}, k_{2} \ldots k_{i}\right)$ where $\left(k_{1} \ldots k_{i}\right)$ are any set of $i$ integers between $i$ and $r$. The generation of these $i$ secondaries at these specific collision points is accompanied by the probability to generate these secondaries at $\left(\bar{p}_{k_{1}} \cdots \bar{p}_{k_{i}}\right)$ given by $\left(a_{k_{1}} \cdot a_{k_{2}} \cdot a_{k_{i}}\right)$, multiplied by the probsbility of not generating secondaries at the remaining (n-i) collision points 
$\left(b_{k+1} \ldots b_{k}\right)$. a is the probability of generating a secondary at $\bar{p}_{s}$; it is a function of both $\bar{r}_{s}$ and $\bar{\Omega}_{s}$ and is given by $\left|\bar{\Omega}_{s} \cdot \bar{n}\right|$ if $\bar{r}_{s}$ is in the volume of integration and zero otherwise. $b_{s}$ is the probability for not generating a secondary at $\left(\bar{p}_{s}\right)$ and is given by $\left(1-a_{s}\right)$. Now we have $i$ secondary particles generated at specific collision points of the primary particle: $\bar{p}_{k_{1}}, \ldots \cdot \bar{p}_{k_{1}}$. Condsider, for example the secondary generated at $\bar{p}_{k_{1}}$. A possible track for that secondary is given by $\left(\vec{p}_{k_{1}}, \bar{p}_{k_{1}}^{\prime}, \bar{p}_{k+1}^{\prime} \cdots \bar{p}_{j_{k l}}^{\prime}\right)$. The first point, $\bar{p}_{k_{1}}$, indicates the enission point of the secondary particle and the primed vectors indicate subsequent collison points of the secondary up to its absorption at $\bar{p}_{j_{k_{1}}}^{\prime}$. In the above uistory there are $\left(j_{k_{1}}-k_{1}\right)$ secondary collision points and when the summation is done (with the integration to consider all possible tracks of that secondary particle) $j_{k_{1}}$ will range from $k_{1}$ to infinity. Thus an independent event includes: a priwary particle's n-collision history with its probability density $f^{\star \star n}\left(\bar{p}_{0} \bar{p}_{1}, \ldots \bar{p}_{n}\right)$; generation of $i$ secondaries with probability $a_{k_{1}} \cdots a_{k_{1}} \cdot b_{k+1} \cdots b_{k_{n}}$; the histories of these $i$ secondaries with probability density

$$
f\left(\bar{p}_{k_{1}}, \bar{p}_{k_{1}}^{\prime} \cdots \bar{p}_{j_{k_{1}}}^{\prime}\right) \cdot f\left(\bar{p}_{k_{2}}, \bar{p}_{k_{2}}^{\prime} \cdots \bar{p}_{j_{k_{2}}}^{\prime}\right) \ldots f\left(\bar{p}_{k_{i}}, \bar{p}_{k_{i}}^{\prime} \ldots \bar{p}_{j_{k_{i}}}^{\prime}\right)
$$

Each secondary particle will make a score denoted by $\omega_{c_{k_{\ell}}}$ with $1 \leq \ell \leq i$, and the total score for the primary particle is then $\left(w_{c_{k_{1}}}+w_{c_{2}}+\ldots+w_{c}\right)$. To obtain averaging over all possible events we sum over $n(1$ to $\infty)$, over $i$ ( 1 to $n)$, over all possible $\left(\begin{array}{l}n \\ i\end{array}\right)$ permutations of $\left(k_{1} \ldots k_{i}\right)$, and over each $j_{k_{\ell}}$ from $k_{\ell}$ to $\infty$ and integrate over all collision points.

It is ciear now that when the sum of the scores is squared it will result in cross terms representing the correlation between secondaries generated from a common source particle. Physically this correlation stems from the fact that these two secondaries have a partial common history which is that part of thia 
history of the primary before the first of the secondaries was generated. Our next step is to neglect these cross terms i.e., neglect the correlation between secondaries, at a later stage we will briefly discuss the effect of this approximation. As a result of this approximation we will now have, in Eq. (19), the sun of squares of the scores rather then the square of the sum. This allows us to rearrange the equation by regrouping terms. If we look at all the teras of the equation for a fixed $n$ then many of them will include $w_{c}^{2}$ (and the same number of terms will include $w_{c_{2}}^{2}$ and $w_{c_{3}}^{2}$ etc. up to $w_{c}^{2}$ ). For exanple, $w_{c_{1}}^{2}$ will appear in a term where $n=5, i=2,\left(k_{1}, k_{2}\right)=(1,3)$ and such a term will contain $\left(w_{c_{1}}^{2}+w_{c_{2}}^{2}\right)$. The idea now is to factor out $w_{c_{1}}^{2}$ (as well as any other $w_{c_{l}}^{2}$ for $1 \leq \ell \leq n)$. Consider any specific $w_{c_{\ell}}^{2}$ and let us observe a typical, though general, term in which it will appear. For a fixed $n$, a fixed $i$ and a fixed $k_{1} \ldots k_{i}$ (one of the $k^{\prime} s$ must equal $\ell$ and we take it to be $k_{1}=\ell$ ) the coefficient of $w_{c}^{2}$ will be:

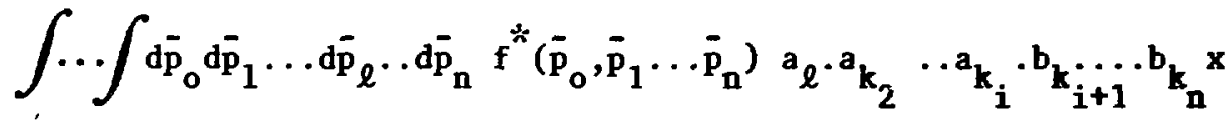

$$
\begin{aligned}
& \int \ldots \int_{j_{\ell}=\ell}^{\infty} \sum_{j_{k_{2}}=k_{2}}^{\infty} \ldots \ldots \sum_{j_{k_{i}}=k_{i}}^{\infty} f^{*}\left(\bar{p}_{\ell}, \bar{p}_{\ell}^{\prime} \ldots \bar{p}_{j_{\ell}}^{\prime}\right) f^{\dot{*}}\left(\bar{p}_{k_{2}}, \bar{p}_{k_{2}}^{\prime} \ldots \bar{p}_{j_{k}}^{\prime}\right) \ldots \\
& \ldots f\left(\bar{p}_{k_{i}}, \bar{p}_{k_{i}}^{\prime} \ldots \bar{p}_{j_{k_{i}}}^{\prime}\right) \bar{p}_{\ell}^{\prime} \ldots d \bar{p}_{j_{\ell}}^{\prime} d \bar{p}_{k_{2}}^{\prime} \ldots d \bar{p}_{j_{k_{2}}}^{\prime} \ldots d \bar{p}_{k_{i}}^{\prime} \ldots d \bar{p}_{j_{k_{i}}}^{\prime}
\end{aligned}
$$

It is important now to observe that except for $f^{*}\left(\bar{p}_{\ell}, \bar{p}_{\ell}^{\prime} \ldots \cdot \bar{p}_{j}^{\prime}\right)$ no other probability density of a secondary carries any score with it. As a result all other probability densities for the other secondaries will sum and integrate to unity. This is so since for these secondaries the sumation and integration will simply yield the probability to have that secondary follow any possible track, given that it was generated at some $\bar{p}_{k}$; this probability is unity (see Eq. (18)). 
Thus the above coefficient will reduce to

$$
\begin{aligned}
& \int \ldots \int d \bar{p}_{0} d \bar{p}_{\overline{1}} \ldots d \bar{p}_{n} f^{\star}\left(\bar{p}_{0}, \bar{p}_{1} \ldots \bar{p}_{n}\right) a_{\ell} \cdot a_{k_{2}} \ldots a_{k_{i}} \cdot b_{k_{i}+1} \ldots b_{k_{n}} x \\
& x \sum_{j_{\ell}=\ell}^{\infty} \int \ldots \int \mathrm{dp}_{\ell}^{-1} \ldots \mathrm{d}_{\mathrm{j}_{\ell}}^{-!} \mathrm{f}^{*}\left(\overline{\mathrm{p}}_{\ell}, \overline{\mathrm{p}}_{\ell}^{\prime} \ldots \overline{\mathrm{p}}_{\mathrm{j}_{\ell}}^{\prime}\right)
\end{aligned}
$$

Note that the sum and integral over $\mathrm{f}^{*}\left(\overline{\mathrm{p}}_{\ell}, \overline{\mathrm{p}}_{\ell}^{\prime} \ldots \overline{\mathrm{p}}_{j}^{\prime}\right)$ will not yield unity since $f^{*}\left(\bar{p}_{\ell}, \bar{p}_{\ell}^{\prime} \cdots \bar{p}_{j_{\ell}}^{\prime}\right)$ is multiplied by $w_{c_{\ell}}^{2}$ which is, in general, a function of all collision points $\overline{\mathrm{p}}_{0}, \overline{\mathrm{p}}_{1} \ldots \overline{\mathrm{p}}_{\ell}, \overline{\mathrm{p}}_{\ell}^{\prime} \cdot \overline{\mathrm{p}}_{\mathbf{j}}$. Now when we group together all the terms in which $w_{c_{\ell}}^{2}$ will appear, for a fixed $n$ we have to consider $i$ varying from $i$ to $n$ and all the possible permutations in which $l$ appears. Looking at the above cuofficient we note that this consideration will concern only the product $a_{\ell} \ldots a_{k_{i}}$ $b_{k_{i}+1} \ldots b_{k_{n}}$. In all the terms to be considered we will have a product of $n$ factors (some of them $a^{\prime} s$ and some $b^{\prime} s$ ) and in all of them $a_{\ell}$ will appear. The sun of all these terms will be

$$
\begin{aligned}
& a_{\ell} \cdot\left\{\prod_{j=1}^{n} a_{j}+\sum_{j=1}^{n} a_{1} \cdots_{j-1} b_{j} \cdots a_{n}+\sum_{j_{1}=1}^{n} \sum_{j_{2}=j_{1}+1}^{n} a_{1} \cdots a_{j_{1}-1} b_{j_{1}} \cdots_{j_{2}} b_{n}\right. \\
& +\ldots \ldots+\prod_{j=1}^{n} b_{j}
\end{aligned}
$$

The prime above each sum and product indicates that the index cannot take the value of $\ell$. Looking at the above expression we see that the first term accounts for the case where for the fixed $n$ primary collision points $n$ secondaries where generated (of which one was generated at $\bar{p}_{\ell}$ ). The second term accounts for (n-1) secondaries generated (of which one at $\bar{p}_{\ell}$ ), so that we sum over $b_{j}$ taking all 
values between 1 and $n$ except $\ell$. This series continues up to the last term in which only 1 secondary was generated at $\bar{p}_{\ell}$. We may now note that the above sum in the curly brackets is unity. This since the sum can be looked upon as the sum of frobabilities of all the possibl, events of a composite binonial experiment composed of (n-1) simple binomial events each of which may have two possible outcomes with probabilities $a_{m}$ and $\left(1-a_{m}\right)$. With these above considerations Eq. (19) can now be written in a rearranged form as

$$
\begin{gathered}
s_{c}^{2}=\sum_{n=1}^{\infty} \sum_{i=1}^{n} \sum_{k_{i}=i}^{\infty} \int \ldots \int d_{p_{0}} \ldots d \bar{p}_{n} \int \ldots \int d \bar{p}_{i}^{\prime} \ldots d \bar{p}_{k_{i}} \\
x f^{*}\left(\bar{p}_{0}, \bar{p}_{1} \ldots \bar{p}_{n}\right) a_{i} w_{c_{i}}^{2} f^{*}\left(\bar{p}_{i}, \bar{p}_{i}^{\prime} \ldots \bar{p}_{k_{i}^{\prime}}^{\prime}\right)
\end{gathered}
$$

where $a_{i}$ is given by $\chi\left(\bar{r}_{i}\right) \cdot\left|\Omega_{i} \cdot \vec{n}\right|$ and $\chi\left(\bar{r}_{i}\right)$ is the volume function, which is 1 inside $V$ and 0 outside.

We may now rewrite Eq. (20) introducing the explicit dependence of the probability densities and the transport kernels:

$$
\begin{aligned}
& s_{c}^{2}=\sum_{n=1}^{\infty} \sum_{i=1}^{n} \sum_{k_{i}=i}^{\infty} \int \ldots \int\left[Q^{*}\left(\bar{\Omega}_{1}\right) T^{*}\left(\bar{\Omega}_{1} ; \bar{x}_{0}+\bar{x}_{1}\right) \ldots \ldots \ldots \ldots T^{*}\left(\bar{\Omega}_{i} ; \bar{x}_{i-1} \bar{x}_{i}\right)\right] x \\
& \cdot\left[\mathrm{T}^{*}\left(\bar{\Omega}_{i} ; \bar{x}_{i} \rightarrow \bar{y}_{i}\right) C^{*}\left(\bar{y}_{i} ; \bar{\Omega}_{i}+\bar{\Omega}_{i+1}^{\prime}\right) \mathrm{T}^{*}\left(\bar{\Omega}_{i+1}^{\prime} ; \bar{y}_{i}+\bar{y}_{i+1}\right) \ldots \mathrm{T}^{*}\left(\bar{\Omega}_{k_{i}}^{\prime} ; \bar{y}_{k}+\bar{y}_{k-1}\right) P\left(\bar{y}_{k_{i}}\right)\right] \\
& {\left[c^{*}\left(\bar{x}_{i} ; \bar{\Omega}_{i}+\bar{\Omega}_{i+1}\right) \ldots T^{*}\left(\bar{\Omega}_{n} ; \bar{x}_{n-1} \rightarrow \bar{x}_{n}\right) p\left(\bar{x}_{n}\right) v_{c_{i}}^{2}\left(\bar{p}_{i}, \bar{p}_{i}^{\prime} \ldots \bar{p}_{k_{i}}^{\prime}\right)\right] \cdot\left|\Omega_{i} \cdot n\right| . \cdot x\left(x_{i}\right)} \\
& d \bar{p}_{1} \quad \ldots d \bar{p}_{n} d \bar{p}_{i}^{\prime} \ldots . d \bar{p}_{k_{1}}^{\prime}
\end{aligned}
$$

Note that primary collision points are now given by $\bar{p}=(\bar{x}, \bar{\Omega})$ whereas those of secondaries by $\bar{p}^{\prime}=\left(\bar{y}, \bar{\Omega}^{\prime}\right)$. In Eq. (21), in the first bracket, the transport of the primary up to the collision point where the secondary is emitted is described, 
within the second bracket the track of the secondary is displayed and in the third bracket the continuation of the random walk of the primary is accounted for. Now interchanging the sumation

$$
\sum_{n=1}^{\infty} \sum_{i=1}^{n} \text { into } \sum_{i=1}^{\infty} \sum_{n=i}^{\infty}
$$

we can integrate and sum over $n, i . e$. over the con ,inuation of the primary track after the collision point at which the secondary was generated, yielding unity. This, since we are only integrating over the probability density that a particle that reached a collision at $\left(\bar{x}_{i} \bar{\Omega}_{i}\right)$ will eventually be absorbed at sone later collision. We are then left with

$$
\begin{aligned}
& s_{c}^{2}=\sum \sum \int \ldots \int Q^{*}\left(\bar{\Omega}_{1}\right) \mathrm{T}^{*}\left(\bar{\Omega}_{1} ; \overline{\mathrm{x}}_{0} \rightarrow \overline{\mathrm{x}}_{1}\right) \ldots \mathrm{T}^{*}\left(\bar{\Omega}_{i} ; \overline{\mathrm{x}}_{\mathrm{i}-1} \rightarrow \overline{\mathrm{x}}_{\mathrm{i}}\right) \mathrm{T}^{*}\left(\bar{\Omega}_{\mathrm{i}} ; \overline{\mathrm{x}}_{\mathrm{i}} \rightarrow \overline{\mathrm{y}}_{\mathrm{i}}\right) \\
& i=1 \quad k_{i}=i \\
& \ldots T^{*}\left(\bar{\Omega}_{k}^{\prime} ; \bar{y}_{k_{i}-1}+\bar{y}_{k_{i}}\right) p^{*}\left(\bar{y}_{k_{i}}\right) w_{c_{i}}^{2}\left(\bar{p}_{0}, \ldots \bar{p}_{i}, \bar{p}_{i}^{\prime} \cdots \bar{p}_{k_{i}}^{\prime}\right)\left|\bar{\Omega}_{i} \cdot \bar{p}\right| x\left(\bar{x}_{i}\right) d \bar{p}_{1} \ldots \\
& d \bar{p}_{i-1} d \bar{p}_{i}^{\prime} \ldots d \bar{p}_{k_{i}}^{\prime}
\end{aligned}
$$

Now as was mentioned in the forwer section we may consider the whole track $\vec{p}_{0} \cdots \bar{p}_{i}, \dot{\alpha}_{i} \cdot \cdots \bar{p}_{k_{y}}^{\prime}$ as a track of a secondary starting from the source. Note that now $\left(\bar{x}_{i}, \bar{\Omega}_{i}\right)$ is not a real collison point since the particle, though entering a collision, can not be either absorbed or deflected, thus it is a "transfer" point. To proceed, we change the notation, denoting all collision points by $\bar{p}=(\bar{y}, \bar{\Omega})$ except for $\bar{p}_{i}$, which will now be denoted by $\bar{q}_{i}=\left(\bar{x}_{i}, \bar{\Omega}_{i}\right)$ again we interchange the sumation 


$$
\begin{aligned}
& \sum_{i=1}^{\infty} \sum_{k_{i}=i}^{\infty} \text { to } \sum_{k_{i}=1}^{\infty} \sum_{i=1}^{k_{i}} \text { and obtain } \\
& s_{c}^{2}=\sum_{k_{i}=1}^{\infty} \sum_{i=1}^{k_{i}} \int \ldots \int Q^{*}\left(\bar{\Omega}_{1}\right) T^{*}\left(\bar{\Omega}_{1} ; \bar{y}_{0} \bar{y}_{1}\right) c^{*}\left(\bar{y}_{1} ; \Omega_{1} \bar{\Omega}_{1}\right) \ldots T^{*}\left(\bar{\Omega}_{i} ; \bar{y}_{i-1} \rightarrow \bar{x}_{i}\right)
\end{aligned}
$$

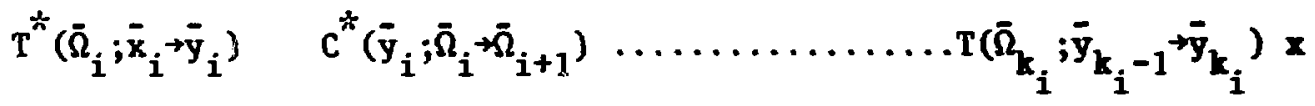

$$
\begin{aligned}
& p\left(\bar{y}_{k_{i}}\right) \times\left(\bar{x}_{i}\right) w_{c_{i}}^{2}\left(\bar{p}_{0}, \ldots \bar{p}_{i-1}, \bar{q}_{i}, \bar{p}_{i} \ldots \bar{p}_{k_{i}}\right) \mid \bar{\Omega}_{i} \cdot \bar{n}_{\mid} d \bar{p}_{1} \ldots d \bar{p}_{i-1} d \bar{q}_{i} d \bar{p}_{i} \cdot d \bar{p}_{k_{i}}
\end{aligned}
$$

Note that as indicated in the former section we transformed the averaging from that over primaries to that over generalized secondaries. Now we can integrate over $\bar{q}_{i}$. Since $\bar{y}_{i-1}, \bar{x}_{i}$ and $y_{i}$ are colinear, the integration over $d \bar{q}_{i}=d \bar{\Omega}_{i} d \bar{x}_{i}$ will be reduced to an integration over $d x_{i}{ }^{*}$ over the line segment between $\bar{y}_{i-1}$ and $\bar{y}_{i}$. Again, now, if in the biased free flight kernel only the total cross section is changed, as is in particular the case with the exponential biasing, we may write

$$
T\left(\Omega_{i} ; \bar{y}_{i-1} \rightarrow \bar{x}_{i}\right) T^{*}\left(\bar{\Omega}_{i} ; \bar{x}_{i} \rightarrow \bar{y}_{i}\right)=\Sigma_{t}^{*}\left(\bar{x}_{i}\right) T\left(\bar{\Omega}_{i} ; \bar{y}_{i-1} \rightarrow \bar{y}_{i}\right)
$$

Inserting the above relation into Eq. (22) we obtain (by comparing to Eq. (9) for those cases in which the volume of integration contains homogeneous media

$$
s_{c}^{2}=\sum_{k=1}^{\infty} \int \ldots \int f^{*}\left(\bar{p}_{0}, \bar{p}_{1} ; \ldots \bar{p}_{k}\right) \Sigma_{t}^{*} d \bar{p}_{1} \ldots d \bar{p}_{k} w_{c}^{2}\left(\bar{p}_{1} \ldots \bar{p}_{k}\right) \quad x
$$

FThe integration over $d \bar{\Omega}_{i}$ and the angular part of $d \bar{x}_{i}$ will yield unity due to the angular delła functions implicit in the free flight kernells $T *\left(\bar{\Omega}_{i} ; \bar{y}_{i-1}+x_{i}\right)$ and $T\left(\Omega_{i} ; \bar{x}_{i} \rightarrow \bar{y}_{i}\right)$ 


$$
x \sum_{i=1}^{k}\left|\bar{\Omega}_{i} \cdot \bar{n}\right| \int_{0}^{-\bar{y}_{i}^{-y_{i-1}} \mid} x\left(\bar{x}_{i}\right) \bar{d} x_{i}
$$

Moreover, for all possible estimators where the estination is done only at collision points in the detector region (which includes all types of transaission probleas) we may use the relation of Eq. (15) and obtain

$$
\begin{aligned}
& s_{c}^{2}=\sum_{k=1}^{\infty} \int \ldots \int f^{*}\left(p_{0}, \bar{p}_{1}, \ldots \bar{p}_{k}\right) w_{p}^{2}\left(\bar{p}_{1} \ldots \bar{p}_{k}\right) d \bar{p}_{1} \ldots d \bar{p}_{k} \cdot \Sigma_{t}^{*} \\
& \left|\overline{\mathrm{y}}_{i}-\overline{\mathrm{y}}_{\mathrm{i}-1}\right| \\
& \text { - }\left[\sum_{i=1}^{\sum_{i}^{k}\left|\bar{\Omega}_{i} \cdot \bar{n}^{\prime}\right| \int_{0}^{\sum_{t}^{*} \Delta^{2}} x\left(\bar{x}_{i}\right) d \bar{x}_{i}}\right] \cdot \frac{\left(\bar{\Omega}_{i} \cdot \bar{n}\right)}{\left|\bar{\Omega}_{i} \cdot \bar{n}\right|}
\end{aligned}
$$

If we neglect now negative secondaries $i . e .$, consider only primaries for which $\left(\bar{\Omega}_{i} \bar{n}\right)$ within the volune of integration is positive then

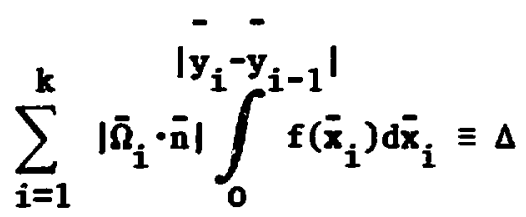

and is indepedent of the integral for all the tracks for which $w_{p}$ is nonzero. Upon comparison to Eq. (12) we thus may conclude the nost important relation

$$
s_{c}^{2}=\frac{s_{R}^{2}}{\Sigma_{t}^{*} \Delta}
$$


At this point we attempt to extend the application of the above desired expression to include also splitting. Splitting and Russian Roullette are commonly used in variance reduction. This may be formulated in the following way. Let a collision point be $\bar{p}_{i}=\left(\bar{x}_{i}, \bar{\Omega}_{i}\right)$ and let the direction with which the particle emerges from that collision be $\bar{\Omega}_{i+1}$, thus the enission point corresponding to $\left(\bar{x}_{i}, \bar{\Omega}_{i}\right)$ is $\left(\bar{x}_{i}, \bar{\Omega}_{i+1}\right)$. At each emission point the particle is "splitted" into $v\left(\bar{x}_{i}, \bar{\Omega}_{i+1}\right)$ particles each of which is then followed independ_atly with its weight divided by $v\left(\bar{x}_{i}, \bar{\Omega}_{i+1}\right)$. Russian Roullete is included in that description by sieply taking $v\left(\bar{x}_{i}, \bar{\Omega}_{i+1}\right)<1$. It is worthwhile to note that splitting is basically not a biasing method, it should be rather viewed as a method that utilizes more detailed averages taken for future contributions irom phase space points which are considered more important. Using this point of view the division of the particle weight by $v$ is not a weight adjustment but more like an averaging factor much in the same sense that source particle contributions are divided by the total number of source particles generated without considering that division as a weight adjustment. Consider now the particle tracks $\left(\bar{p}_{0}, \bar{p}_{1} \ldots \bar{p}_{n}\right)$ where $p_{0}$ is the source point and $\vec{p}_{1} \cdots \bar{p}_{n}$ are collision points. If the probability density of such a track is $f *\left(\bar{p}_{0}, \bar{p}_{1} \cdots \bar{p}_{n}\right)$ then with the introduction of splitting the probability density will be

$$
f_{s}^{*}\left(\bar{p}_{0}, p_{1}^{-} \ldots, \bar{p}_{n}\right)=f^{*}\left(p_{0}, \bar{p}_{1} \ldots, \bar{p}_{n}\right) \cdot \prod_{i=1}^{n-1} v\left(\bar{r}_{i}, \bar{\Omega}_{i+1}\right)
$$

and the weight of the particle entering the collision at $\bar{p}_{n}$ is then multiplied by

$$
\left[\prod_{i=1}^{n-1} v\left(\bar{r}_{i}, \bar{\Omega}_{i+1}\right)\right]^{-1}
$$

Inserting these expressions into Eq. (21) we obtain

$$
s_{c}^{2}=\sum_{n=1}^{\infty} \sum_{i=1}^{\infty} \sum_{k=1}^{\infty} \int \ldots \int\left[Q^{*}\left(\bar{\Omega}_{1}\right) T^{*}\left(\bar{\Omega}_{1} ; \bar{x}_{0}+\bar{x}_{1}\right) v\left(\bar{x}_{0} \bar{\Omega}_{1}\right) c^{*}\left(\bar{x}_{1}, \bar{\Omega}_{2}\right) T^{*}\left(\bar{\Omega}_{2} ; \bar{x}_{1} \cdot \bar{x}_{1}\right)\right.
$$




$$
\begin{aligned}
& \left.\ldots c^{*}\left(\bar{x}_{i-1} ; \bar{\Omega}_{i-1}+\bar{\Omega}_{i}\right) v\left(\bar{x}_{i-1}, \bar{\Omega}_{i}\right) T^{*}\left(\bar{\Omega}_{i} ; \bar{x}_{i-1} \rightarrow \bar{x}_{i}\right)\right] T^{*}\left(\bar{\Omega}_{i} ; \bar{x}_{i} \rightarrow \bar{y}_{i}\right) \\
& C^{*}\left(\bar{y}_{i} ; \bar{\Omega}_{i}^{\prime}+\bar{\Omega}_{i+1}^{\prime}\right) v\left(\bar{y}_{i}, \bar{\Omega}_{i+1}^{\prime}\right) \ldots T^{*}\left(\bar{\Omega}_{k_{i}}^{\prime} ; \bar{y}_{k_{i}-1} \rightarrow \bar{y}_{k_{i}}\right) P^{*}\left(\bar{y}_{k_{i}}\right) \\
& {\left[\prod_{j=1}^{i-1} v\left(\bar{x}_{j}, \bar{\Omega}_{j+1}\right) \cdot \prod_{j=1} v\left(\bar{y}_{j}, \bar{\Omega}_{j+1}^{\prime}\right)\right]^{-2} \cdot\left[c^{*}\left(\bar{x}_{i}^{\prime}, \bar{\Omega}_{i} \rightarrow \bar{\Omega}_{i+1}\right) v\left(\bar{x}_{i}, \bar{\Omega}_{i+1}\right) \ldots\right.} \\
& \left.\ldots T^{*}\left(\bar{\Omega}_{n} ; \bar{x}_{n-1} \bar{i}_{n}\right) \bar{p}^{*}\left(\bar{x}_{n}\right)\right] \cdot \prod_{j=1}^{n-1} v\left(\bar{x}_{j}, \bar{\Omega}_{j+1}\right) w_{c_{i}}^{2}\left(\bar{p}_{i}, \bar{p}_{i}^{\prime} \ldots \bar{p}_{k_{i}^{\prime}}^{\prime}\right) \mid \bar{\Omega}_{i} \cdot \bar{n}_{\mid} \cdot x\left(\bar{x}_{i}\right) \\
& d \bar{p}_{1} \ldots d \bar{p}_{n} d \bar{p}_{i}^{\prime} \ldots d \bar{p}_{k_{i}}^{\prime},
\end{aligned}
$$

where the notation and meaning of each variable is the same as in Eq. (21). Repeating now the same steps taken in the last section in Eqs. (22) through (24) it is straight-forward to see that Eq. (26) is still vaiid.

In those problems that are of most interest (deep penetration, streaming in pipes, etc.) the second moment is much larger than the average (resulting in a large fractional error which requires a large sample of bistories for the calculation). We may then safely approximate the variance by the second moment. To account for the average time per particle we take the following view. Let the average time per particle in regular Monte Carlo be $\tau_{R}$. Now the average time per particle in the contributon scheme will be given by $\tau_{c}=\tau_{R}+N_{\text {sec }} \cdot \tau_{s}$ where $N_{\text {sec }}$ is the average number of secondaries produced per primary and $\tau_{s}$ is the average time per secondary. Usually $\tau_{s}<\tau_{R}$ since the secondaries are produced deep inside the medium. We nay write $\tau_{s}=\left(\tau_{s} / \tau_{R}\right) \cdot \tau_{R}=\beta \cdot \tau_{R}$ where

$$
\beta<1, \text { also } N_{\text {sec }}=\frac{N_{s}}{N_{p}} \text {, }
$$


where $\mathrm{N}_{s}$ is the total number of secondaries and $\mathrm{N}_{p}$ is the number of primaries used in the calculation. Since secondaries are produced at collision points in the volume of integration with probabilty $|\bar{\Omega} \cdot \bar{n}|$ we may write

$$
N_{\text {sec }}=\iint_{V}|\bar{\Omega} \cdot \bar{n}| \Psi(\bar{r}, \bar{\Omega}) \mathrm{dV} d \bar{\Omega}
$$

where the integration extends over the volume of integration and $\psi(\overline{\mathbf{r}}, \bar{\Omega})$ is the collision rate. Finally we may now write the expression for the benefit factor

$$
B=\frac{q_{C}}{q_{R}}=\frac{S_{R}^{2} \tau_{R}}{s_{c}^{2} \tau_{c}}=\Sigma \Sigma_{t}^{*} \cdot \Delta \cdot\left(1+\beta \frac{N_{s}}{N_{p}}\right)^{-1}
$$

In the case of exponential path stretching where $\Sigma_{t}^{*}=\Sigma_{t} \cdot(1-p)$ we obtain

$$
B=\frac{{ }^{q_{C}}}{q_{R}}=\Sigma_{t} \cdot \Delta \cdot(1-p)\left(1+\beta \frac{N_{s}}{N_{p}}\right)^{-1} .
$$

V. DISCUSSION ON THE BENEFIT FACTOR

Let us first list the approximations and assumptions involved in the theory and their impact on the practicai use of Eqs. (28) and (29).

a. Correlation terms between secondaries generated from one primary particle are neglected. Thus the theory will be most valid in those cases where each primary particle generates only one secondary. The larger the number of secondaries produced per primary, the larger will be the deviation of the theory from reality.

b. Negative secondaries, i.e. those secondaries generated at collision sites for which $\bar{n} \cdot \bar{\Omega}<0$, are neglected. Both the effect of the negative secondaries and the correlation will tend to decrease the benefit and make the theory unreliable, this does not mean that a benefit may not be expected in such cases, only that in such problems Eq. (28) provides an upper limit to the benefit. It 
may be worthwhile mentioning that the effect of these approximations will be particularly small in the class of "pipe-like" problems, i.e., streaming in pipe geometry. In such cases $\Delta$ may be chosen very large thus yielding a large benefit. Since the volume of integration may then be relatively thin (radially), the probability of a negative secondary to get to the detector and the probability of a primary to generate more than one secondary that will reach the detector are small. These considerations imply that one may expect the contributon Monte Carlo method to be most fruitfull in this class of probiems, this point is further varified by the results presented in the next secticn.

c. Within the volume of integration the total cross section $\Sigma_{t}^{*}$ is assumed to be constant. This will generally not be true in energy dependent cases. Though the energy variable was not istroduced in the derivation of the theory it can be incorporated without any difficulties merely by setting $\bar{p}_{i}=\left(\bar{x}_{i}, \vec{\Omega}_{i}, E_{i}\right)$. However this will mean that to derive the expression for $s_{c}^{2}$ averages must be taken over the energy spectrum in Eq. (23), a prohibitively difficult task. This difficulty can be avoided by the following simple procedure. Let $\Sigma_{t \ell}^{* \frac{2}{n}}$ be the lowest value the total cross section assumes over the energy band considered, then one way zenerate the secondaries with an extra probability $\Sigma_{t \ell}^{*} / \Sigma_{t}^{*}(E)$ and multiply their weight by $\Sigma_{t}^{*}(E) / \Sigma_{t \ell}^{*}$. If this Russian Roulette procedure is introduced into $\mathrm{Eq}$. (22) it is easy to see that it will yield

$$
\frac{\mathrm{s}_{\mathrm{R}}^{2}}{\mathrm{~s}_{\mathrm{c}}^{2}}=\sum_{\mathrm{tl}}^{\stackrel{\ell}{*}} \cdot \Delta,
$$

independent of the energy dependence of the cross section. This can be liziful if $\Sigma_{t \ell^{*}}^{*} \Delta$ remains larger than one. Alternatively one may use this procedure with some parameter $\Sigma$ which is not necessarily the lower bound of the cross sections; this can be used to moderate sharp changes in the cross sections in resonance regions. Results pertainings to energy dependent cases are beyond the scope of this paper and will be reported in the near future.

d. The theory applies to all estimators that score information upon the particles reaching the detector region, for other estimators, like the uncollided flux estimator, the new method cannot be applied in its presenc form. 
e. The theory is valid for any biasing of the collision kernel, ${ }^{\top}$ that includes angular biasing, importance sampling and weighting in lieu of absorption. It also includes exponential path stretching, phase space splitting and Rusミian Roulette. Note however, that biasing techniques, and splitting in particular, may drastically change the number of secondaries generated, so that, even though the variance ra:io may not change beyond the effect of the correlation and the negative secondaries, the benefit factor may change due to changes in the time ratio.

There are few more aspects of the theory which are worth mentioning. One may note that the exponential path stretching is the only biasing method to have an influence on the variance ratio. We believe that this is because no other biasing method changes the relation between the collision spectrum of the two methods (Regular and Contributon Monte Carlo) and the Heumann series structure of the problem ${ }^{(8)}$. For an extreme exampie, consider the case that the stretching parameter $p$ is taken to be unity, thus $\Sigma_{t}^{*}=0$. In such a case in regular Monte Carlo only the zero'th Neumann series term will be estimated, however the Contributon method will yield no estimate at all since secondaries will not be generated. Indeed, an increasing missmatch between the collision spectrun and the Neumann spectrum ${ }^{++}$may be responsible for the decrease of the variance ratio for increasing $p$.

As to the question of the choice of the volume of integration, the theory, Eq. (29), indicates that the variance ratio $S_{R}^{2} / S_{c}^{2}$ increases linearly with the lateral dimension, $\Delta$, but is independent of the location of the volumn of integration in the medium. Yet the time ratio,

$$
1+\beta \frac{\mathrm{N}_{\mathrm{s}}}{\mathrm{N}_{\mathrm{p}}}=\frac{\mathrm{T}_{\mathrm{c}}}{\mathrm{T}_{\mathrm{R}}}
$$

is dependent both on $\Delta$ and on the location of the volumn. Clearly between the two effects, that of the variance ratio and that of the time ratio dependence on $\Delta$, one may expect an optimum value for $\Delta$, say $\Delta_{0}$. Whereas the optimum $\Delta$ may require some experinental search ${ }^{+++}$, the location of the volume of integration, for any given $\Delta$, should be chosen as far as possible from the source to minimize the number of generated secondaries and thus minimize the time ratio.

This should be evident since in the derivation a biased collision kernel c* was used without specifying any particular biasing scheme. 
When vacuum voids exist in the volume of integration the contributon method must be slightly modified. Since in a void there are no collisions, the contribution of the flux within the void to the total response will be missing. To account for this the following procedure must be adopted: As soon as a primary particle enters the void a track length estimate of that particle is scored. The particle leaving the void now serves both as a primary and as a secondary, and for the contributon estimate its score upon reaching the detector is multiplied by $(\bar{\Omega} \cdot \bar{n})$ and by its track length estimate.

To the best of our knowledge the above outlined theory presents for the first time a general analytic expression for the variance ratio of two Monte Carlo methods. This was made possible due to the simple relation between the weight of a secondary and the weight of the corresponding primary (not the primary that generated the secondary but a primary who's track is identical to that of the secondary). The theory is not intended to give exact values of the benefit factor, except for those cases in which all the above outlined assumptions are valid, a situation which does not exiscs in most p! actical cases. Rather, the theory in meant to give some insight into the method and to provide the potential user a better and non-heuristic tool for deciding when the use of the new method is beneficial. This is done in the following way: $\Delta$ is defined prior to the calculation, $\Sigma_{t}^{*}$ is either constant or may be taken as the smallest value of the cross section or as an average (or one may use the $\Sigma_{t l^{*}}^{*} / \Sigma_{t}^{*}(E)$ procedure). Thus the approximate value of $\left(\Sigma_{t}^{*} \Delta\right)$ can be defined prior to the calculation. A short sample run can give the time ratio $T_{R} / T_{c}$ and the product $\Sigma_{t}^{*} \Delta \cdot T_{R} / T_{c}$ gives an upper bound to the expected benefit. It is important at this point to mention an additional most important feature of the "Contributon method". The introduction of the method to a given code does not interfere with the transport of the primary particles in any way. Thus together with the estimate ob-

++ The $k^{\prime}$ th term of the collision spectrum is defined as the relative number of particles that scored in the detector region after making $k$ collisions in the medium, thus contributing to the estimate of the $k^{\prime}+i$ Neumann series term. The $k^{\prime}$ th term of the "Neumann spectrum" is the ratio of the $k$ 'th term of the Neumann series to the sum of all terms.

+++ Our studies indicate that usually the benefit factor is a slowly varying function of $\Delta$ around the optimum. Thus a crude estimate of $\Delta$ is sufficient in most cases. 
contained with the new method one still obtains the results of the regular Monte Carlo, for any chosen biasing scheme. This feature is unique to the contributon method and is most important, especially in difficult situations where one is forced to use low accuracy estimates due :o time limitation. In such cases it is not uncommon to encounter a situation of a low statistically estimated standard deviation together with an estimate for the target response which may sonetimes differ by as much as an order of magnitude from the correct average. In such problems, it is most useful to obtain for a small penalty in the calculational time, an additional almost independent more accurate estimate with the contributon method. The implementation of the new method to a given code is simple and straightforward since basically the transport of the secondaries is identical to that of the primaries; thus existing transport subroutines can be used. Apart from that, one needs only a simple routine to generate the secondaries and a negligible amount of additional storage to store the estimates of the secondaries. Once the new method has been implemented its exclusion (in those cases when the benefit factor is less than one) is trivial.

In the next section results are presented in verification of the above described theoretical considerations.

\section{NUMERICAL RESULTS}

In order to verify and check the above discussed theoretical model a variety of test cases were calculated. The basic geometry used is that of a homogenuous disk of radius $R$ and thickness $z_{0}$ surrounded by vacuum with a $2 \pi$ isotropic point source on the center $(r=0)$ of the $z=0$ surface. In all cases transmission through surfaces were considered. The surface detectors considered were: (a) a large detector, consisting of transmission through the whole surface at $z=z_{0}$, (b) a small detector consisting of transmission through a small circular detector of radius $R_{d}$ centered on the surface $z=z_{0}$, and (c) a side detector for transmission through the circular surface of the disk $(r=R)$. In all the cases exponential biasing was used such that particles going towards the detector surface are biased with $\Sigma_{t}^{*}=\Sigma_{t}(1-p)$ whereas those that go in an apposite direction are not biased at all. Such biasing dependence is commonly used in transmission problems $^{(8)}$. Weighting in lieu of absorption was used to all collision points and Russian Roulette cutoffs were used to avoid negligible low-weight time-consuming events. Isotropic scattering is assumed with no angular biasing. In each case 
exactly the same biasing was applied to the Regular Monte Carlo and the Contributon Monte Carlo. Experimental efficiency factors were derived from the actual computer runs by estinating the second moment and multiplying it by the average time per source particle. The ratio of the efficiency factors so obtained is the experimental benefit factor $B$ exp. For the predicted or theoretical benefit factor of $\mathrm{Eq}$. (29) values of $\mathrm{N}_{\mathbf{s}}$ needed to be defined from short runs usually with a sample of about $1 / 20$ of the sample required for a reliable estimate of the target response $I$. The parameters to be varied are, $z_{o}, R, p, c=\Sigma_{s} / \Sigma_{t}, \Delta_{t}$, and the detector configuration. The theory was tested against the variation of all these parameters.

In order to compensate for the overestimations introduced in the theory by neglecting correlations and contributions from negative $\bar{n} \cdot \bar{\Omega}^{\prime} s, \beta$ of Eq. (29) was taken to be unity in all cases.

Large Detector: In Fig. 2 we consider a disk of depth 20 mean free paths (m.f.p.) and radius 4 m.f.p., the scattering probability of the medium is $c=$ $\Sigma_{s} / \Sigma_{t}=0.8$ and $p=0.5$. The volume of integration is taken to be the volume between two surfaces $z=z_{1}$ and $z=z_{2}$ such that $\Delta=z_{2}-z_{1}$ and $\bar{n}$ is pointing at all points in the $z$ direction. The $z=z_{2}$ surface is taken to coincide with the $z=z_{0}$ surface (also the detector surface) as recomended by the theory (to place the volume of integration as far as possible from the source to avoid large collision densities). This type of volume of integration was used for all the large detector test cases to follow. The surface $z=z_{1}$ was varied such that $\Delta$ was changed from $\Delta=2 \mathrm{~m}$.f.p to $\Delta=20 \mathrm{~m} . \mathrm{f} \cdot \mathrm{p}$. Good agreement between the theoretical and the experimental results is evident in Fig. 2. Indeed we see that there is an optimum $\Delta=15$. The solid straight line indicates the expected behavior of $s_{R}^{2} / s_{c}^{2}$ with $\Delta$ where the effect of the time ratio is not included. At low values of $\Delta$, when the number of secondaries produced is small and, as a result, $T_{c}$ is amost equal to $T_{R}$, the benefit factor is tangential to $S_{R}^{2} / S_{c}^{2}$ and increases almost linearly with $\Delta$. At higher values of $\Delta, T_{c}$ increases at a much faster rate than the second moments ratio due to the non-linear increases in $\mathrm{N}_{s}$ and an optimum is reached. For comparison purposes, results are shown also for splitting. The splitting used was such as to prociuce the same number of secondaries in a given $\Delta$, and the same exponential bias and weighting in lieu of absorption was used in the splitting scheme. It is important to note that this 


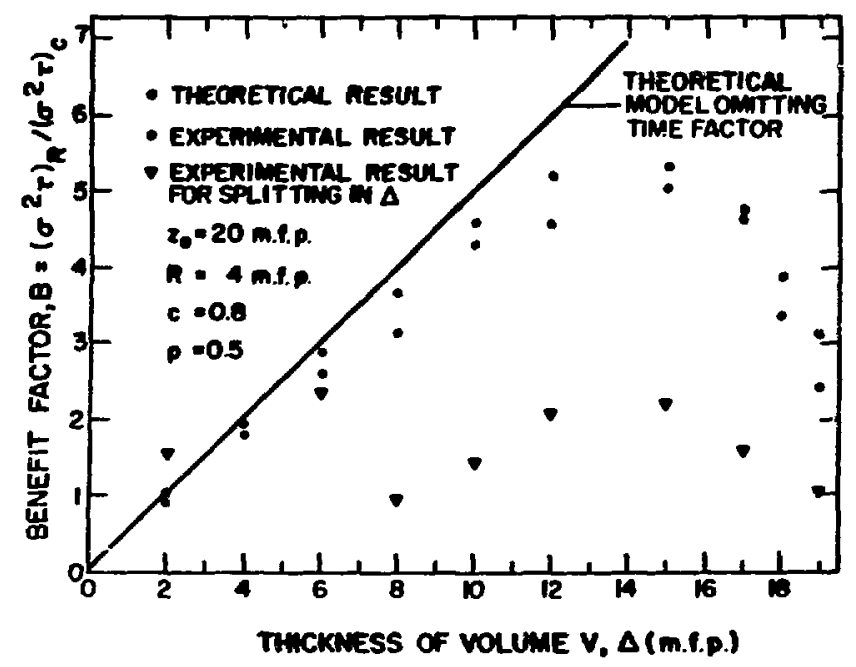

Fig. 2. Transmission through 20 m.f.p. disk.

was not meant to be a comparison between splitting and the contributon schene. No attempt was made to optimize the splitting since the splitting is done only in the volume $V$ and in a sense it is a randon splitting whare secondaries are not splitted any further. The sole purpose of this was to create as, " secondaries as are created by the contributon method in order to denonstrate. at the benefit obtained in the contributon scheme is not a result of the generation of secondaries but of the special new recipe used. In fact, as explained in the introduction, since splitting is a biasing scheme while the contributon method uses a new recipe to compute detector responses, the only valid comparison is one where splitting is applied in the same way, both, to regular Monte Carlo and the to contributon scheme. A few remarks concerning such a comparison will be made in the conclusions.

In Fig. 3 a sample case in the geometry of Fig. 2 is shown for $\Delta=10$. The behavior of the detector response estimates as function of the number of histories is displayed. It is interestin to note that successive contributon nethod estimates reach the assumed exact value with a relatively sall number of histories 
and once reaching that, the statistical fluctuations around it are snaller than would be expected from the variance. This behavior was observed in other cases tested; it may result from the fact that possibly the exact fistribution of the scores is not a normal distribution. In Fig. 4 the paraneter varied is the discs radius $R$, the thickness of the disc is 10 m.f.p., $\Delta$ is kept constant at 5 a.f.p. where again $z_{2}=10=z_{0}$ and the radius of the disc is varied frow $2 \mathrm{~m}$.f.p. to 12 m.f.p. It is seen that as the radius of the disc increases, the difference between the theory and the experiment increases from about $5 \%$ to $35 \%$. Equation (29) tends to over-estimate the benefit when $R$ increases. This is clearly due to the negative contributons and the correlations neglected in the theory. In Fig. 5 again $R$ is varied for the 20 m.f.p. disc penetration. However this time both $\Delta$ and $p$ are also varied. The variation of $\Delta$ was not aimed to optimize results but rather to keep the number of negative secondaries relatively sall as $R$ increases. In comparison to $\mathrm{Fig} .4$, it is seen that the benefit obtained for 20 m.f.p. (at $R=4$ and 6 m.f.p.) is about twice as large as that obtained at $10 \mathrm{m.f.p.,} \mathrm{as} \mathrm{predicted} \mathrm{by} \mathrm{the} \mathrm{theory.} \mathrm{Again,} \mathrm{the} \mathrm{agreement} \mathrm{between} \mathrm{the} \mathrm{theory}$ and the experiment in Fig. 5 is satisfactory. Another feature of the contributon method is displayed in Fig. 5. As was pointed out in the introduction, the transport of the primary particliss is not affected by the generation of the secondaries, which means that the primaries can be used to give a separate estimate, which is exactly equal to the estimate obtained from the regular Monte Carlo. This separate estimate can be combined with the estinate obtained by the contributon method in a linear combination of the form $\xi_{c s}=\xi_{s} \cdot d+(1-d) \xi_{p}$, where $\xi_{s}$ and $\xi_{p}$ are the estimates due to only secondaries and only prinaries respectively, and $d$ is the linear combination parameter. The combination must not be done for each source particle, instead one may accumulate the separate average estimates and combine them in the form $\left\langle\xi_{c s}\right\rangle=d\left\langle\xi_{s}\right\rangle+(1-d) \cdot\left\langle\xi_{p}\right\rangle$. In this case one must also tally the average product $\left\langle\xi_{s} \xi_{p}\right\rangle$ to estimate the correlation for the estimation of the covariance of $\xi_{\mathrm{cs}}$. This form is convenient since it makes it possible to scan at the end of the run over values of $d$ in order to obtain the optimal $d$ to be used. Such a linear combination of results is shown in Fig. 5 for $d=0.5$. It is interesting to note that as long as the benefit factor is bigger than 2 it is beneficial to use $d=1$ or else a reduction in the benefit is caused because the estimates from the primaries alone are inferior to those of the secondaries alone; however, for benefits below 2 an improvenent 


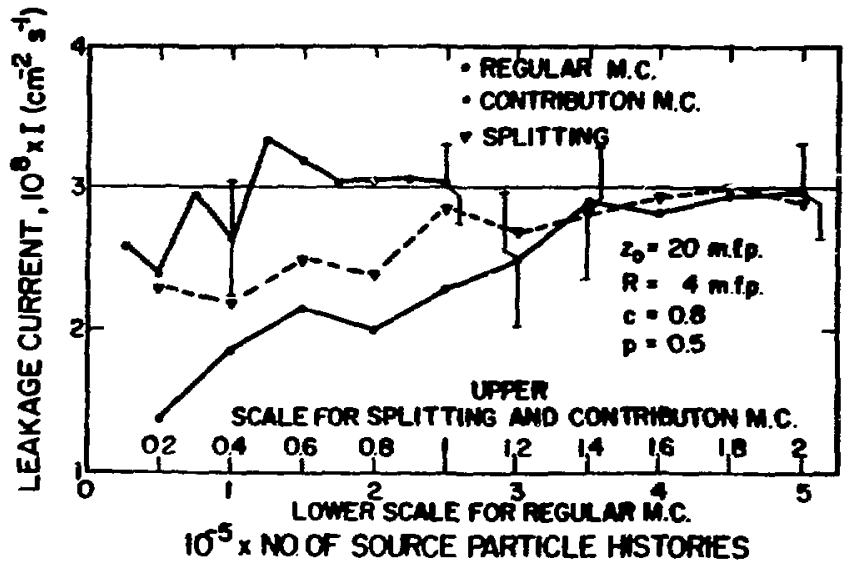

Fig. 3. Disk transmission through 20 m.f.p.

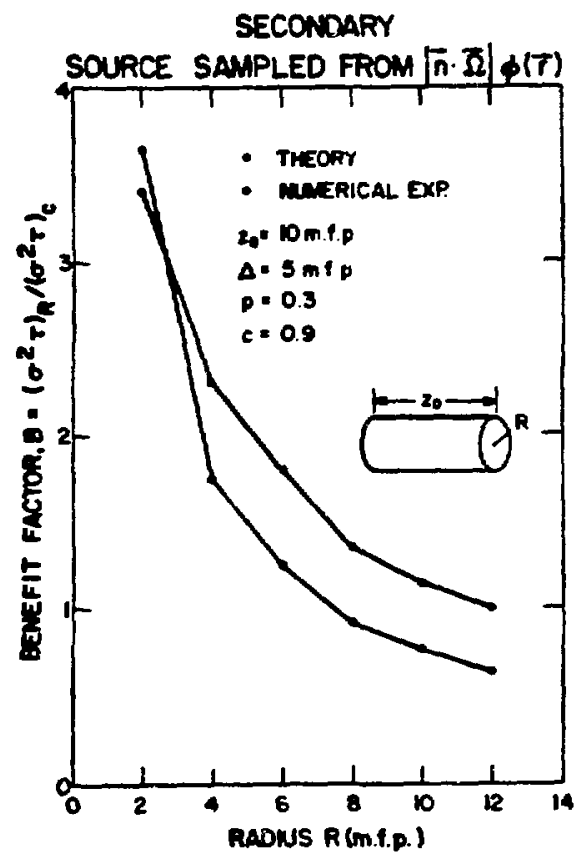

Fig. 4. Disk transmission through 10 m.f.p. 
below 2 an improvement may be obtained by the use of the linear combination. This statement was found to be generally true for all the test cases calculated. In general, it is always advisable to use this combination since it requires a negligible additional time and it can provide, sometimes, optimized estimates (with smaller variance than its components).

In Fig. 6 the dependence of $s_{R}^{2} / s_{c}^{2}$ on $p$ is tested with all other parameters held fixed while $p$ is changed form 0.1 to 0.8 . The solid line presents the linear behavior predicted by the theory and the experimental result seems to follow very well the theoretical prediction. In Fig. 7 the prediction that $s_{c}^{2}$ is independent of the position of the volume of integration is checked. In this case $\Delta=4 \mathrm{m.f.p}$. is held fixed and $z_{1}$ is moved from $z_{1}=1$ to $z_{1}=11$, and this for $z_{0}=15 \mathrm{~m} . \mathrm{f} . \mathrm{p}$. penetration. It is seen that as the volime of integration is brought closer to the source the second moment (in the figure the relative standard deviation is shown) remains constant, within the statistical fluctuations, whereas the efficiency of the computations is systematically decreasing due to the increase of $\mathrm{N}_{\mathrm{s}}$. It is also clear that the best resuit is obtained when the volume is taken as far as possible from the source just as predicted by the theory.

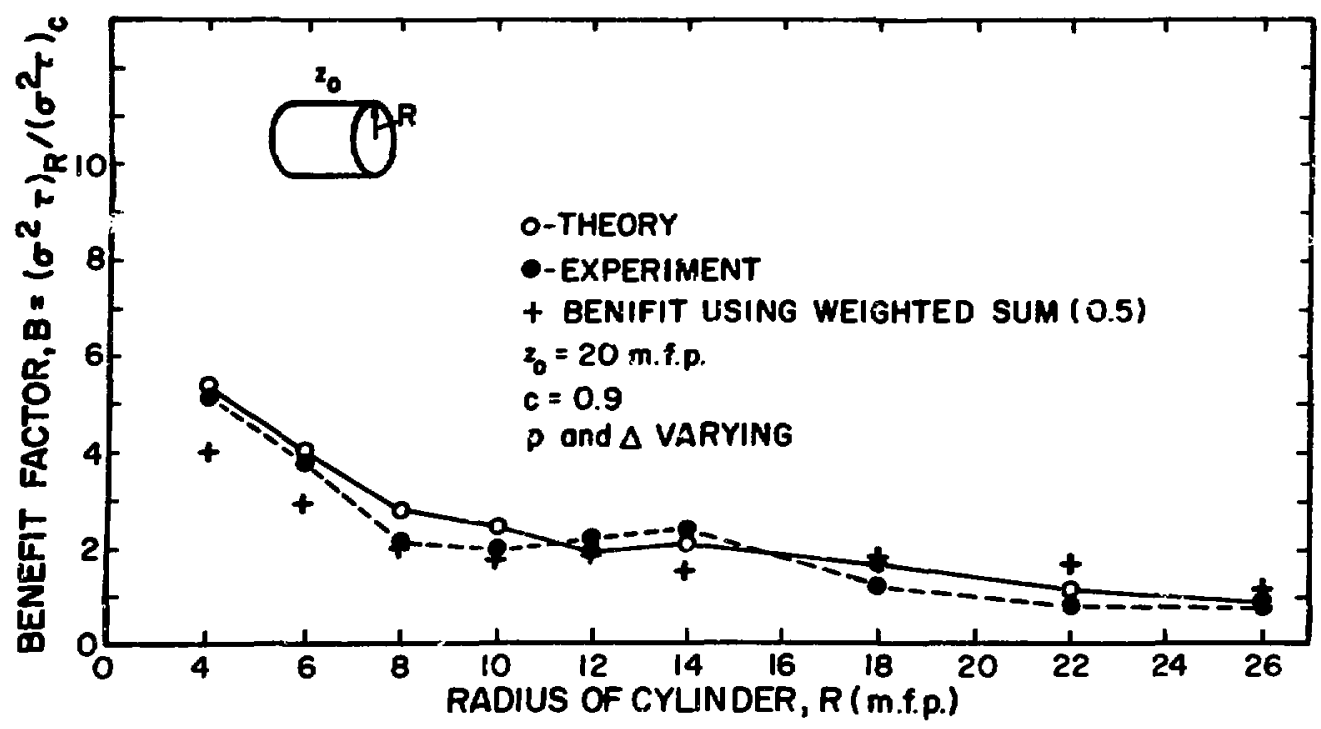

Fig. 5. Disk transmission through 20 m.f.p. 


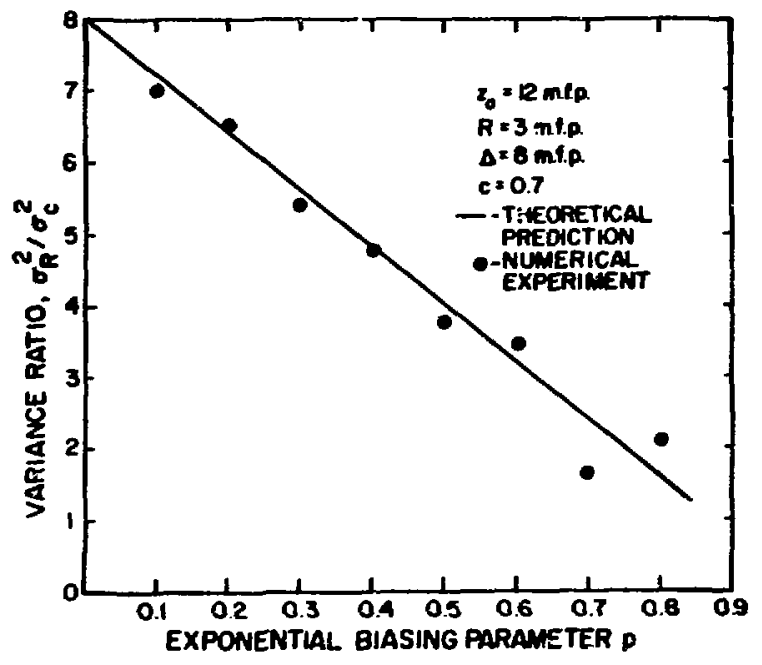

Fig. 6. Disk transmission through 12 m.f.p.

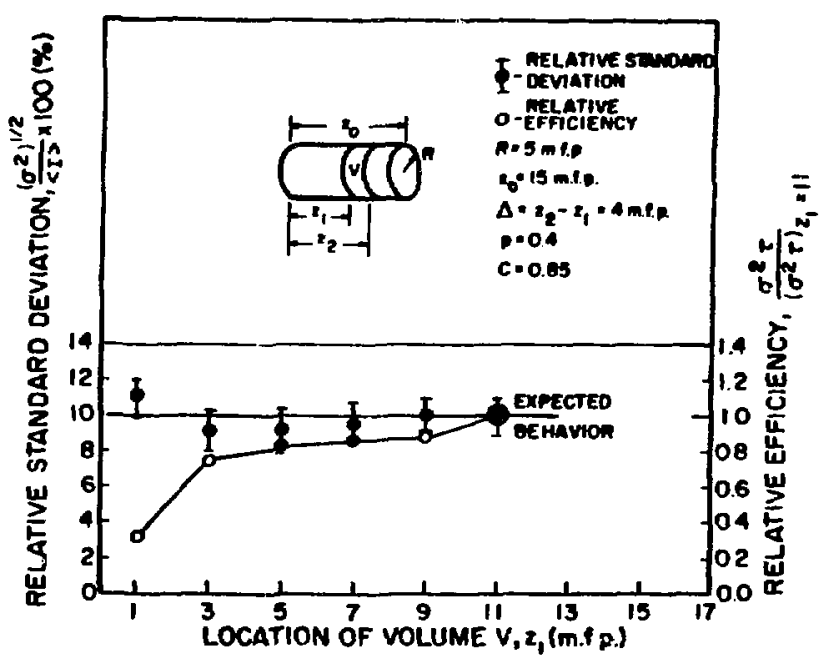

Fig. 7. Disk transmission through 15 n.f.p. 
In Figures 8 and 9 results of the various estimates as a function of the number of histories are shown. The benefit of the contributon method is clearly seen in terms of a smaller variance and again the less fluctuating behavior of the cortributon method is demonstrated.

Side Detector: In Fig. 10 the basic geometry is again the disk as described before. However now the transmission through the circular surface $(r=R)$ is the target response. The varying parameter $i s ~ R$ which now expresses not only the depth of the penetration, but also the fact that the shape of the volume of integration is radically changed. We cannot anymore use the volume $V$ as used for the large detector configuration since that configuration for $V$ will not separate the source from the detector region. $V$ is now taken to be a volume contained between two cylindrical surfaces $r=r_{1}$, and $r=r_{2}$ such that $\Delta=r_{2}-r_{1}$. Again we take $r_{2}=R$ to optimize the position of the volume of integration and $\vec{n}$ is pointing towards the detector. In this case $\Delta$ and $p$ were varied to obtain near optimum values for each $R$; the thickness of the disk is held constant at 4 m.f.p. As expected, with increasing depth of penetration, the benefit increases up to a factor of $\cong 6.8$ and the agreement between the theory and the experiment is very good. In Fig. 11 the estimates, as a function of the number of histories, are shown for the side detector of Fig. 10, for $R=15$ ( $B \cong 5$ ). The relatively smooth and fast converging characteristic of the contributon estimate is demonstrated.

Small detector: This is a most interesting case in which the flexibility of the volume of integration and the "natural" angular biasing in the contributon scheme are demonstrated. The detector, the current through which is the target response, is now a circular disk centered around the $z$ axis on the $z=z_{2}$ surface of radius $R_{d}$. Note that the volume of integration which was used for the large detector case is in principle valid also for shis configuration, however in that volume $\bar{n}$ will not be pointing at the detector for every point in the volume for which $r>R_{d}$. Therefore we consider now the following volume. Let $z_{s}$ be a point on the $z$ axis (whose origin is at the source point) and consider two spherical suzisces concentric about $z_{s}$. Let the radius of the small sphere be $R_{1}=\left[R_{d}^{2}+\right.$ $\left.\left(z_{s}-z_{o}\right)^{2}\right]^{\frac{1}{2}}$ (where $z_{s}>z_{0}$ and outside the disk) such that the detector region is exactly that part of the surface $z=z_{0}$ which is enclosed by the small sphere. The second, larger sphere has a radius $R_{2}$ given by $R_{2}=R_{1}+\Delta$ where $\Delta$ is fixed. We take the volume of integration to be the intersection of the shell between the two spheres and the disk, thus $\Delta$ is the lateral dimension of the volume. Now for $z_{s}=z_{0}$ the common center of the spheres lays on the detector and at every point 


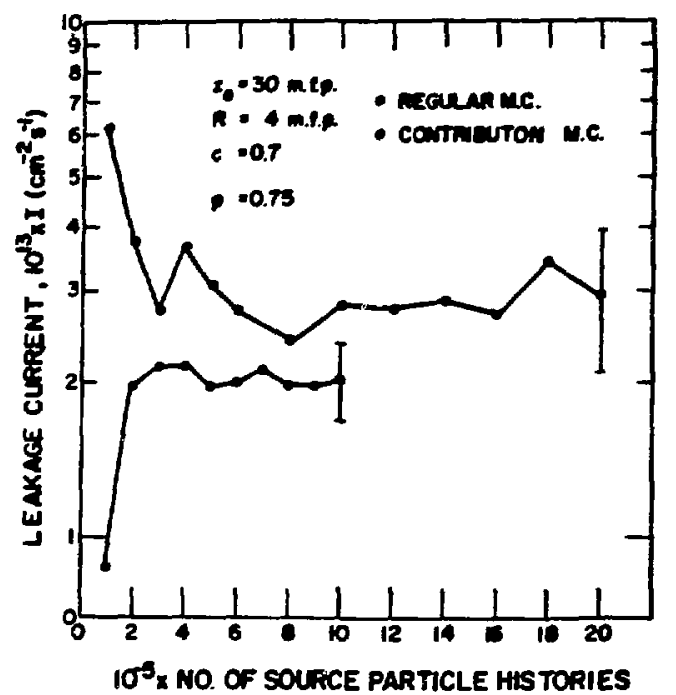

Fig. 8. Disk transmission through 30 m.f.p.

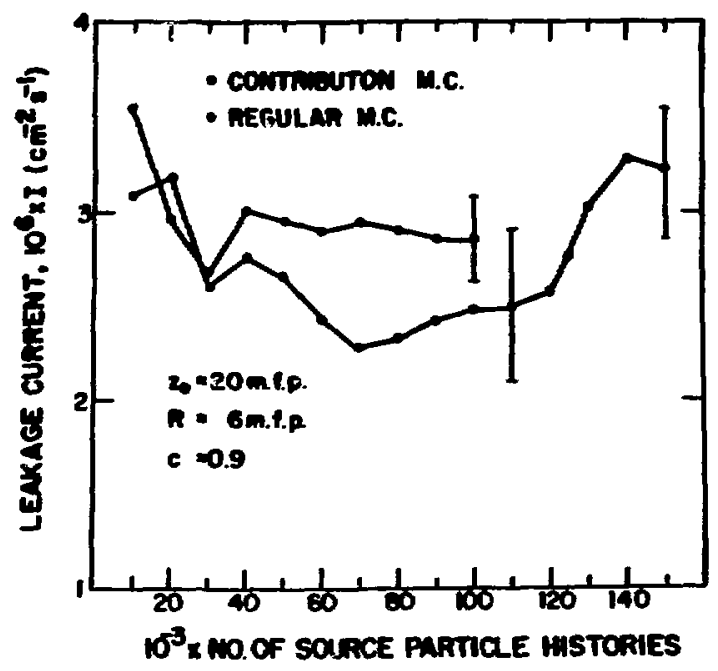

Fig. 9. Disk transwission through 20 m.f.p.

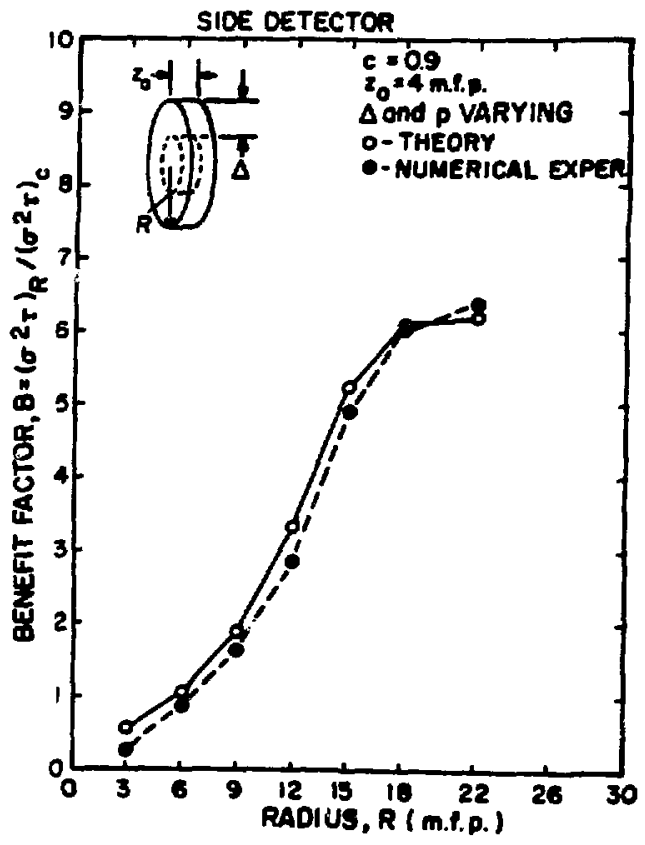

Fig. 10. Disk with side detector.

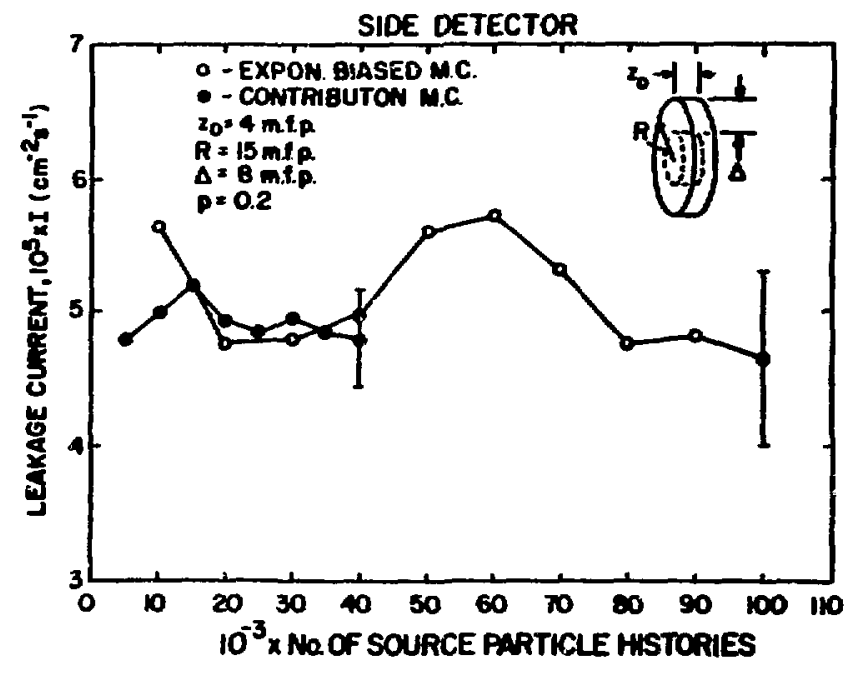

Fig. 11. Disk with side detector. 
within the volune of integration $\overline{\mathbf{n}}$ will be directed towards the detector. For such a volune maximal angular biasing is obtained in the sense that secondaries directed toward the detector are generated with largest probability, $|\overline{\mathbf{n}} \cdot \bar{\Omega}|$ is maximal. On the other hand, we may note that between the small sphere and the detector, there is a region (a volune cut out of the small sphere by the intersection of the surface $z=z_{0}$ and the small sphere) which is close to the detector (thus of high importance) and in which secondaries are not generated because it is outside the volume of integration. This region is characterized by a curvature parameter X (see Fig. 12) given by $R_{1}-\left(z_{s}-z_{0}\right)$; this region is a "dead" volue, as far as the generation of secondaries is concerned. As 2 goes to infinity lwo opposing things will happen: (1) the curvature of the spheres will decrease so that the angular biasing due to the $|\bar{n} \cdot \bar{\Omega}|$-proportional saapling of secondaries becomes less effective, such that eventually for $z_{s}>z_{0}$ this spherical volume of integration will coincide with the flat volume used for the large detector yielding the smallest angular biasing towards the small circular detector. (2) On the other hand, when $z_{0}$ goes to infinity the "dead" volume will decrease (indicated by a decrease in $X$ ) enabling the volume of integration to generate secondary particles closer to the ietector, from where they have a better chance of scoring. Between these two opposing effects we expect an optimum for the benefit with respect to $z_{s}$ (for a given $\Delta$ ) in thi range $z_{0}<z_{s}<\infty$. Also we expect $s_{c}^{2}$ to remain unaffected by the change of $z_{s}$ since, $s_{c}^{2}$ depends on $\Delta$ only. In the specific case shown in Figs. 12 and 13 the parameters used are $R=15$ m.f.p., $R_{d}=3$ m.f.p, $z_{o}=15 \mathrm{~m} . f \cdot p$ and $\Delta=5 \mathrm{~m} . f . p$. A clear maximum in the benefit is shown in Fig. 12 at $z_{s}=19$ (with a considerable large dead volume of $X=1$ m.f.p). The agreement between the theoretical and experimental results is very good. In Fig. 13 the behavior of $s_{c}^{2}$ (in the figure percentage standard deviation is shown) as $z_{s}$ is changed is displayed. It is easily seen that $s_{c}^{2}$ is fairly constant with $z_{s}$ as predicted. For comparison, the stacistical fluctuations in $S_{R}^{2}$ are also shown for the same number of independent runs.

In Fig. 14 a sumary of a large number of cases is shown, including those displayed in the preceding figures and others that were not displayed, with a variety of choices of the various parameters involved. The theoretical berefit as predicted by Eq. (29) is the horizontal axis and the experimental result the vertical axis. If the theory would have been exact, all points would lay on the solid line, however this scatter diagram indicates that most of the points concentrate around this line indicating that for the cases tested the theory does 


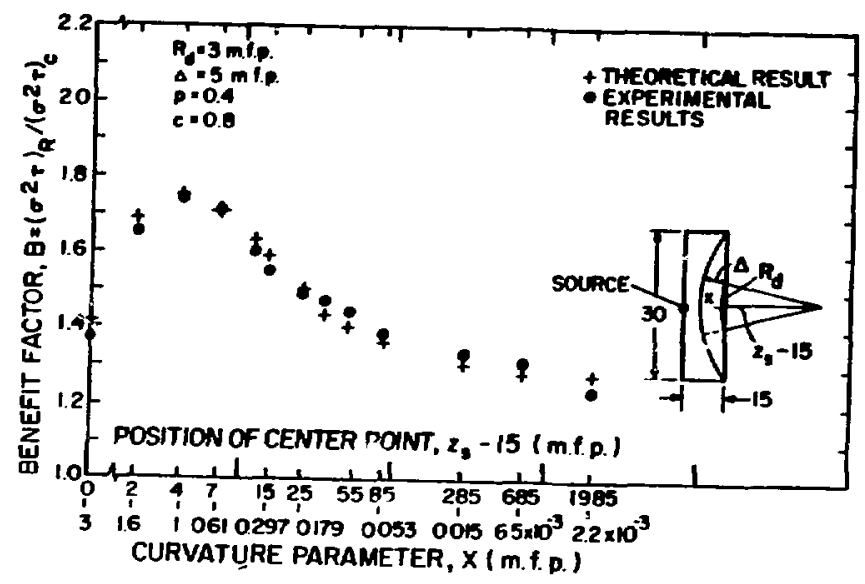

Fig. 12. Disk with small leakage detector.

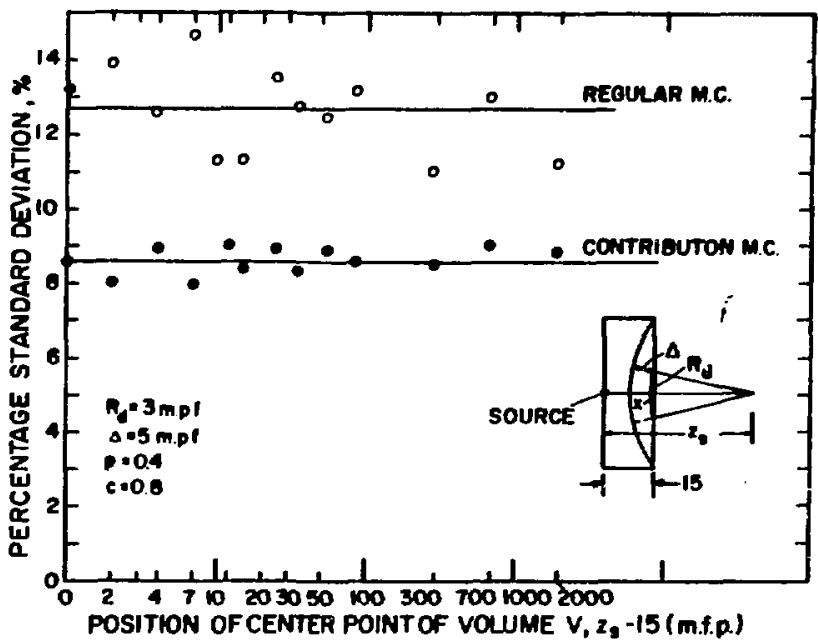

Fig. 13. Disk with small leakage detector.

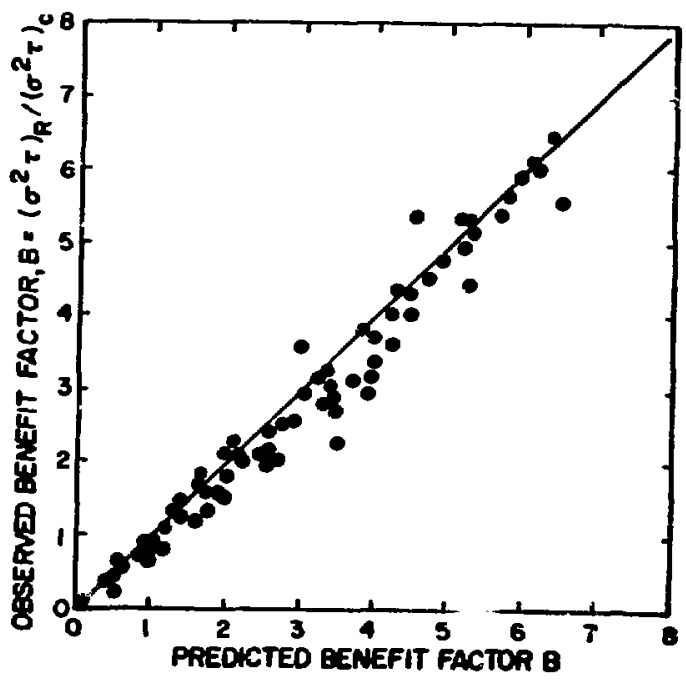

Fig. 14. Theory vs. numerical experiment for 70 independent cases. 
predict satisfactorily the reality. Fig. 14 also indicates that for most cases the theory will slightly overestimate the benefit.

Finally, ve display in Table I results obtained by implementing the new method into the Los Alamos multipurpose photon-neutron transport code MCNP ${ }^{9}$. The problem consists of a $3 \mathrm{~m}$ long bare sodium pipe of $1 \mathrm{~m}$ diameter. The target response is again the leakage current at the end surface of the pipe due to a normal $14 \mathrm{MeV}$ surface source at the inlet of the pipe. Space splitting was used in the following way: the pipe is divided into 5 cells by 4 planar surfaces. To each cell ( $k$ ) an importance integer number is assigned $I(k)$. As the particle crosses the surface between cells $k$ and $k+1$ the particle is split into $I(k+1) /$ $I(k)$ particles. Exponential path length stretching is also used in the form $\Sigma_{t}^{*}=\Sigma_{t} \cdot(1-p \cdot u)$ where $u$ is the cosine of the angle between the particle's direction and the direction of the symmetry axis of the pipe. The leakage current was considered only in the energy interval 13.5-14 $\mathrm{MeV}$, thus the calculation may be considered mono-energetic. Heighting in lieu of absorption is also used with weight cut-offs to avoid low-weight time-consuming events. In Table I results are displayed for four cases: Three in which the amount of splitting is changed (everything else kept constant) and in th s last case no splitting and no exponential path length stretching was used (anaiog $M$. C., except for the weighting in lieu of absorption). For all cases $\Delta$ was taken to be $150 \mathrm{~cm}$ and attached $t$, thr detector surface. In each case $2 \times 10^{4}$ source particles were used for the cc..tributon M.C. and $6 \times 10^{4}$ source particles for the Regular M.C. First we note that considerable improvement is obtained with the use of the contributon M.C. The benefit factors may be obtained simply by multiplying the variance ratios by the time ratio (which was determined experimentally). Note that increasing the amount of splitting affects the results in two ways as is seen from the Table. First, due presumably to the increased correlation effect the deviation between the predicted variance ratio and the experimental one are increased and, secondly, increased splitting increases considerably the time ratio. A severe splitting, especially within the volume of integration, may drastically increase the time ratio by generating inuch more secondaries then splitted primaries. This leads to the conclusion that whenever splitting is used one should first optimize the regular Monte Carlo with respect to splitting and then verify whether or not contributon Monte Carlo should be used for that given splitting scheme. 
TABLE I

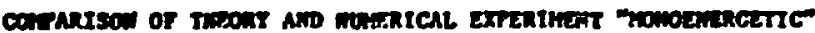

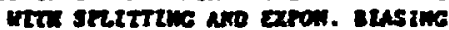

\begin{tabular}{|c|c|c|c|c|c|c|c|c|}
\hline \multirow{2}{*}{$\begin{array}{l}\text { Splitting } \\
\text { Paramecere }\end{array}$} & \multirow{2}{*}{$\begin{array}{l}\text { Expon. } \\
\text { Bles. }\end{array}$} & \multicolumn{2}{|c|}{$3\left(10^{-4} \mathrm{n} / \mathrm{cm}^{2}=\right)$} & \multicolumn{2}{|c|}{$\sigma_{R}^{2} / \sigma_{C}^{2}$} & \multirow[b]{2}{*}{ Exwer. } & & \\
\hline & & Rerulas M. & Contertb. N. C. & Exprer. & Tivenry & & Exner. & Theors: \\
\hline 12260 & 0.3 & $3.75 \pm 2.55$ & $3.03 \pm 2.52$ & 2.16 & $3.1 \%$ & 1.35 & 1.74 & 2.38 \\
\hline 12224 & 0.3 & $3.85 \pm 3.317$ & $3.91 \pm 3.65 x$ & 2.59 & 3.11 & $1 .: 7$ & 2.2 & 2.72 \\
\hline 12222 & 0.3 & $3.85 \pm 4.178$ & $3.90 \pm 3.97$ & 2.78 & 3.12 & 2.15 & 2.6 & 2.82 \\
\hline 11111 & 0.0 & $3.56 \pm 20.38$ & $3.79 \pm 15.57$ & 4.45 & 4.45 & 2.02 & 6.65 & 4.3 \\
\hline
\end{tabular}

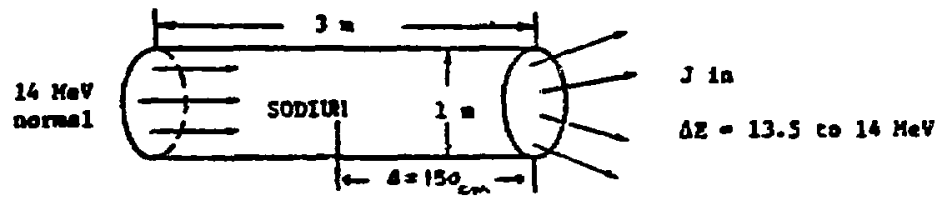

60000 Source Partiela Historfes for zegular M.C.

20000 Source Perzlede Hetordes tor Contributon M.C.

\section{CONCLUSIONS}

A theoretical model was developed to enable an assessment of any benefit to be expected from the use of the contributon method versus regular Monte Carlo with identical biasing used for both methods. The theoretical model was tested against experimental results and was found to be in fairly good agreement with the computer experiments for the test cases. The indication of this study is that especially for the class of "pipe-like" problems advantages of factors of 3-8 and possibly more may be obtained by the use of the new method. The theory is applicable to exponential biasing combined with any type of collision kernel bias. In spite of the fact that the results described here are encouraging, they are not meant to clain ultimate practical advantages of the contributon method in realworld practical shielding and streaming problems. Such a claim can be made only after a large number of practical problems have been solved by independent users and some common experience has been gathered. The results of this study merely point out that it may be worthwile to gather this experience. In an ongoing study we are attempting to take this step and to assess the practicability of the contibuton method on more realistic problem applications. 


\section{REFEREMCES}

1. S. A. W. Gerstl, "A New Concept for Deep-Penetration Transport Calculations and Two New Forms of the Neutron Transport Equation," Los Alamos Scientific Laborato 4 y report LA-6628-MS (1976).

2. M. L. Willians and W. W. Engle, Jr., Nucl. Sci. Eng., 62, 92 (1977).

3. A. Dubi, S. A. W. Gerst1, and Donald J. Dudziak, Nucl. Sci. Eng. 68, 19 (1978).

4. A. Dubi and S. A. W. Gerst1, Trans. An. Nucl. Soc. 30,588 (1978).

5. 0. L. Deutsch and I. L. Carter, Proc. Fifth Int. Conf. Reactor Shielding, Knoxville, Tenn., April 18-23, 1977, CONF-770401, Science Press, 580 (1977).

6. W. M. Stacey, Jr., "Variational Methods in Nuclear Physics," Academic Press, N.Y. (1974).

7. L. S. Abbot and F. R. Mynatt, eds., "Review of ORNL Radiation Shielding Analyses of the Fast Flux Test Facility Reactor," ORNL-5027 (1975).

8. J. Spanier and E. M. Gelbard, "Honte Carỉs Principles and Neutron Transport Problems," Addison-Wesley Publ. Co., Inc. (1969).

9. "MCXP-A Teneral Purpose Monte Carlo Code for Neutron and Photon Transport," Group TD-6, Los Alamos Scientific Laboratory report LA-7396-MS (1978). 